\title{
Genesis and geotectonic setting of podiform chromitites from the Zhob Valley Ophiolite, Pakistan: inferences from chromite composition
}

\author{
${ }^{1}$ Centre of Excellence in Mineralogy, University of Balochistan, Quetta, Pakistan; *Corresponding author, E-mail: ayoub.khan57@yahoo.com \\ ${ }^{2}$ Department of Geoscience, Aarhus University, Høegh-Guldbergs Gade 2, DK-8000 Aarhus C, Denmark \\ ${ }^{3}$ Department of Geological Engineering, Bülent Ecevit University, 67100 Zonguldak, Turkey \\ ${ }^{4}$ Balochistan University of Information Technology, Engineering and Management Sciences, Quetta, Pakistan \\ ${ }^{5}$ National Centre of Excellence in Geology, University of Peshawar, Peshawar, Pakistan
}

(Received: January 16, 2020; Revised accepted: May 9, 2020)

https://doi.org/10.18814/epiiugs/2020/020065

Zhob valley ophiolite comprises Khanozai, Muslim Bagh, and Zhob chromitite occurrences located in Pakistan. These occurrences occur in massive, disseminated and nodular which exhibit both magmatic and deformational texture. The Muslim Bagh and Khanozai chromitites are classified into high-Cr chromitite (Cr\#=0.66-0.85) while Zhob chromitite falls into high-Al chromitite $(\mathrm{Cr} \#=0.53-0.58)$ composition. The calculated values of $\mathrm{Al}_{2} \mathrm{O}_{3}(\mathrm{wt} \%)$ and $\mathrm{TiO}_{2}(\mathrm{wt} \%)$ for parental melts of high-Cr chromitites show similarities with boninitic melts, whereas those of the high-Al ones exhibit MORB melt affinity. Minor and trace element contents (Ti, V, Ni, Zn, and Ga (ppm)) are higher in high-Al chromitites than high-Cr chromitites and contrasting geochemical behavior of these elements with $\mathrm{Cr} \#$ might be due to magma fractionation. MORB normalized major and trace element patterns of high-Cr chromitite show similar patterns with boninite while high-Al chromitite differs from boninite. Field and textural investigations, major and trace element geochemistry, and the presence of hydrous silicate inclusions, indicate that high-Cr chromitite were generated in equilibrium with boninitic melt, possibly in supra subduction zone. In contrast, high-Al chromitite crystallized from MORB-like melt during reaction with depleted mantle and probably originated in a back arc basin tectonic environment.

\section{Introduction}

The origin and nature of geotectonic setting of podiform chromitites is still not fully understood. The chromite composition is largely dependent on the nature and degree of partial melting of the mantle source. Therefore, the chromite composition can be helpful to deter- mine the parental magma composition (Rollinson, 2008) and the geodynamic environment (Dick and Bullen, 1984; Arai et al., 2006; Cai et al., 2012; Ghosh et al., 2014). For instance, high-Cr and high-Al chromitites are considered to be crystallized from boninitic magmas and mid oceanic ridge basalt (MORB) like tholeiitic melts, respectively (Maurel and Maurel, 1982; Melcher et al., 1997; Kamenetsky et al., 2001; Arai et al., 2011; Zaccarini et al., 2011). Furthermore, suprasubduction zone and back-arc basin are the two most important geodynamic environment which influence the geochemical compositions of podiform chromitites (Roberts, 1988; Nicolas, 1989; Arai and Yurimoto, 1994; Zhou and Robinson, 1997; Ballhaus, 1998; Uysal et al., 2007a, b). Chromite is also a useful petrogenetic indicator to determine the composition of the primary mantle source.

The recent development of the techniques regarding the in situ analysis, like Laser Ablation-Inductively Coupled Plasma-Mass Spectrometry (LA-ICP-MS), enable us to analyze a complete suite of minor and trace elements ( $\mathrm{Sc}, \mathrm{Ti}, \mathrm{V}, \mathrm{Mn}, \mathrm{Co}, \mathrm{Ni}, \mathrm{Zn}$, and Ga). Variations in the absolute concentrations of these minor and trace elements in chromian spinel are helpful to determine the composition of parental melt of chromitites and their geotectonic setting (Pagé and Barnes, 2009; Pagé et al., 2012; Colás et al., 2014; González-Jiménez et al., 2015). These elements are very sensitive to temperature and oxygen fugacity conditions prevailing during mantle melting (González-Jiménez et al., 2015; Kapsiotis et al., 2018).

In this study, we present petrographic description of chromitites and the first major $(\mathrm{Cr}, \mathrm{Al}, \mathrm{Fe}, \mathrm{Mg}$ ) and minor-trace element ( $\mathrm{Sc}, \mathrm{Ti}$, $\mathrm{V}, \mathrm{Mn}, \mathrm{Co}, \mathrm{Ni}, \mathrm{Zn}$, and $\mathrm{Ga}$ ) concentrations in chromitites from the Zhob valley ophiolite. We purpose to determine the genesis of Zhob valley chromitite deposits including their parental melt composition and tectonic environment.

\section{Regional Geological Setting}

The rocks of the Zhob valley ophiolite belt and surrounding area 

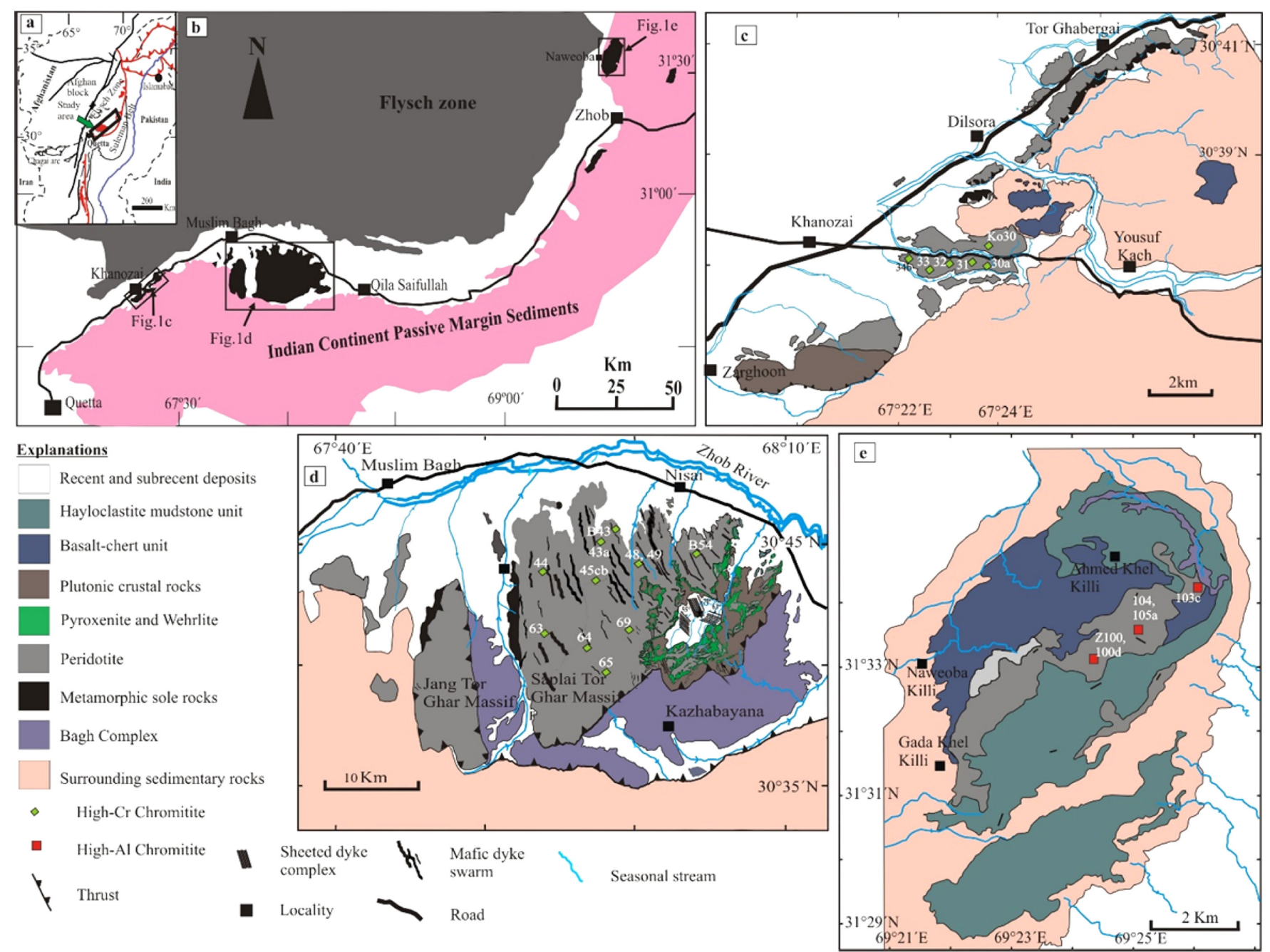

Figure 1. (a) Location map, (b) geological map of Zhob valley ophiolite and Western Sulaiman Fold and Thrust Belt (c) Khanozai ophiolite, (d) Muslim Bagh ophiolite and (e) Naweoba Block of Zhob ophiolite. Sample locations are shown and same symbols will be used for high-Cr and high-Al chromitites in all diagrams. (modified after Bannert et al., 1992).

can broadly be divided into three geologic zones (Fig. 1a, b). In the south, the Calcareous zone is tectonically the lowermost zone of the Indian continental plate margin. This zone contains sedimentary formations that range in age from Early Triassic to Paleocene. These successions are dominantly composed of limestone followed by shale with some sandstone and conglomerate that are deposited in pelagic to shallow marine environments (Hunting Survey Corporation (HSC), 1960). The Calcareous zone is thrusted over by the Suture zone; lying between the Indian Plate and Afghan Block (Gansser, 1979) and consisting of the Muslim Bagh ophiolite and Bagh complex. The detailed descriptions of Muslim Bagh ophiolite and Bagh complex are given in their respective sections below. The third geological zone is the Flysch zone which lies to the north of the Suture zone (Fig. 1b). The stratigraphy of the Flysch zone, revised by Kasi et al. (2012), comprises limestone at the basal part which is succeeded by thick flysch sediments composed of cyclic successions of shale, siltstone and sandstone with locally minor conglomerates. These units are largely deltaic and fluvial in nature and range from Eocene to Pleistocene in age.

\section{Geology of Zhob Valley Ophiolite}

Pakistan contains an extended belt of ophiolites in the Bela-Zhob valley-Waziristan zone from south to north. Bela ophiolite is the largest ophiolite in Pakistan which begins from Bela town (near Arabian Sea coast) up to Khuzdar city while the Zhob valley ophiolite belt extends from northeast of Quetta up to Zhob town, followed by the Waziristan ophiolite further north. The Bela-Zhob valley-Waziristan ophiolite belt demarcates a suture zone between the Indian continental plate and the Afghan Block in northwestern Pakistan (Sengor, 1987). The Zhob valley ophiolitic belt consists of the Khanozai, Muslim Bagh and Zhob ophiolitic bodies (Fig. 1b). Among these bodies, the Khanozai ophiolite comprises both mantle peridotite and crustal mafic-ultramafic cumulates which are underlain by mélange (Fig. 1c; Popal et al., 2018; Ulhaq, 2018). The mantle peridotite covers about two third of the area of the Khanozai ophiolite. The Muslim Bagh ophiolite is well-developed and the largest body in the Zhob valley ophiolite. This ophiolite was described by Vredenburg (1901) and Bilgrami (1964) as intrusive complex, but it was identified by Rossman et al. (1971) as ophiolite 
complex. It contains a nearly complete ophiolitic succession (Penrose, 1972), with a less developed crustal section overlying a thick residual mantle section (Fig. 1d). The crustal portion is composed of ultramafic to mafic cumulate rocks which gradually grades into mafic cumulate or gabbros and poorly developed sheeted dykes at the top (Kakar et al., 2014). The mantle section consists of harzburgite, dunite and lherzo-
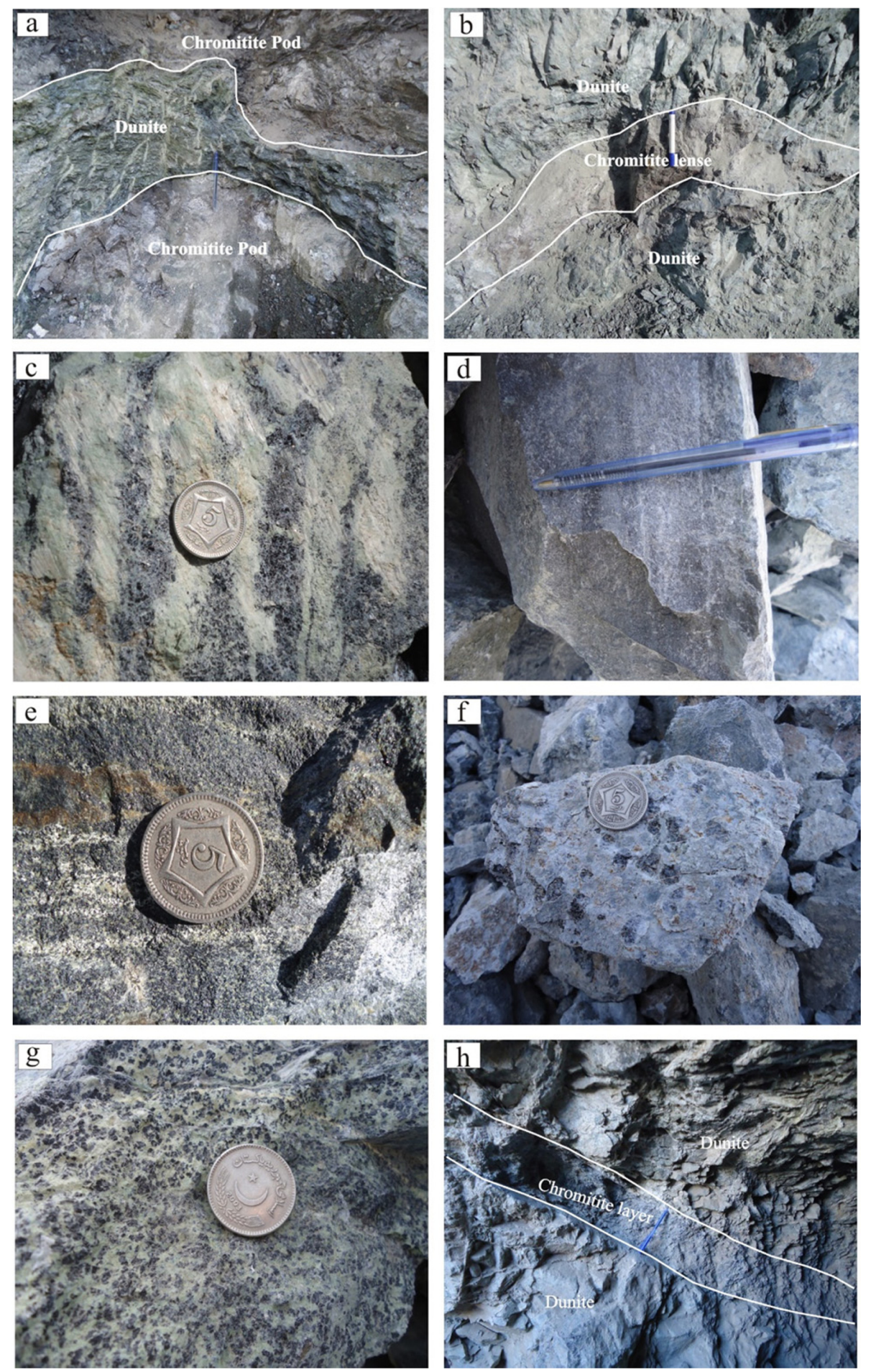

Figure 2. Photographs of Zhob valley chromitites, (a) Chromitite pods with massive textures and sharp contacts with dunite envelopes, (b) chromitites lenses in dunite envelope, (c) banded chromitites, (d) massive texture in Zhob Valley chromitites, (e) chromitites with semi-massive texture, (f) chromitites with nodular textures, (g) disseminated texture in chromitites, (h) layered structure in Zhob valley chromitites. The coin is $27.5 \mathrm{~mm}$ in diameter. lite (Mahmood et al., 1995). These rocks are partially to completely serpentinized (30 to 100\%) and intensively sheared (Mahmood et al., 1995). The swarms of 3 to $15 \mathrm{~m}$ thick mafic dyke cross cut the mantle section of this body (Kakar et al., 2015).

Ahmad (1974), Otsuki et al. (1989) and Sawada et al. (1992) proposed a mid-oceanic ridge tectonic setting for the Muslim Bagh ophiolite. But Hoshino and Anwar (1989), Siddiqui et al. (1996) and Kakar et al. (2014) disagreed and suggested that the Muslim Bagh ophiolite developed in a back arc basin or supra-subduction setting. Furthermore, the Zhob ophiolite consists of three detached bodies around Zhob town namely Naweoba, Omzha, and Ali Khanzai Blocks. Triassic-Jurassic rocks of the Calcareous zone surround all the three bodies (HSC, 1960). The Omzha Block contains mostly two elongated but parallel peridotite bodies. They are up to $3 \mathrm{~km}$ in length and $300 \mathrm{~m}$ in width, and diorite covers the margin of these bodies. The Ali Khanzai Block consists of peridotite and volcanic rocks (HSC, 1960 named it as Bahlolzai). Further, the Naweoba Block contains a thick unit of volcanic rocks with some gabbroic rocks. It also contains serpentinized peridotite which is largely composed of harzburgite and dunite (Ayoub, 2014; Fig. 1e).

\section{Zhob Valley Chromitites}

Chromitite deposits occur in the mantle portion of the Zhob valley ophiolite which is largely composed of highly serpentinized harzburgite and dunite. Chromitite bodies form lenses, pods and banded structures (Fig. 2a, b, c). At many places, these structures do not show any apparent directional relationship with the boundaries of intrusive while at some places chromitite layers are parallel with and have same to dip with host rocks (Bilgrami, 1963, 1969). Pods reach up to $4 \mathrm{~m}$ in thickness while lenses of chromitite are found up to $25 \mathrm{~cm}$. The pods and lenses are surrounded by dunite envelopes which are highly serpentinized. Dunite envelopes make sharp to slightly gradual contact with harzburgite. Chromitites form alternate layers with dunite of varied thickness. Chromitites form massive to semi-massive, nodular, disseminated, and layered or banded textures. Massive chromitite and semi massive chromitite contains aggregates of medium to coarse, subhedral to anhedral grains of chromite (Fig. 2d, e). Furthermore, nodular chromitite is observed at some locations (Fig. 2f) which is massive in nature and has anhedral grain shape. The nodules reach in size up to $10 \mathrm{~mm}$ and are embedded in serpentinized matrix. Disseminated chromitite is 
made up of fine grained subhedral chromite grains (Fig. 2g). Layering in the Zhob valley chromitite is also observed locally in which chromites usually form disseminated texture (Fig. 2h).

\section{Petrography}

Lherzolite forms thin layers at the base of the ophiolitic sequence. The layers are less serpentinized and show porphyroclastic to mylonitic textures. Modal composition of lherzolite is olivine (70\%), clinopyroxene (14\%), orthopyroxene (14\%) and spinel (2\%) (Kakar et al., 2013). Harzburgite shows serpentinization, is generally medium to coarse grained, granoblastic and hypidiomorphic textures. It contains orthopyroxene (25-35\%), serpentine (56-60\%, mostly after olivine), olivine (5-10\%) and chrome spinel (3-6\%) (Siddiqui et al., 1996). Dunite is the most important rock type of the mantle section and it is highly serpentinized across all bodies. It shows granoblastic texture and in some samples the relict core of olivine is surrounded by a serpentinized mesh. Dunite is composed of olivine (15-20\%), serpentine (65-70\%), diopside (3-5\%) and spinel (2-3\%) (Siddiqui et al., 1996). Serpentinites in the Muslim Bagh body of the Zhob valley ophiolite contain $90 \%$ serpentine with minor opaque oxides and olivine. It is massive and greenish in color (Mahmood et al., 1995).

The investigated chromitites from Zhob valley ophiolite are massive-semi massive, disseminated, banded, and nodular in texture. Massive-semi massive chromite grains are surrounded by alteration product such as serpentine (Fig. 3a, b). Also, massive chromitites show brecciated textures in which grains are fragmented in small pieces (Fig. 3c). However, disseminated chromitites contain largely subhedral grains embedded in serpentinized matrix (Fig. 3d).

Chromite is largely unaltered, at the same time a few grains show alteration (Fig. 3e). The ferritchromite alteration occurs along fractures and boundaries of some chromite grains, the development of ferritchromite is not limited to fractures and grain margin, and covers up to $75 \mathrm{vol} \%$ of chromite grain. The formation of ferritchromite yields spongy textures in chromite grains (Fig. 3e).

Anhydrous-hydrous silicates, and sulfide minerals occur in chromitite samples as inclusions and matrix in chromite grains. Olivine is the most frequent silicate phase that occurs as matrix among chromite grains as well as inclusions (Fig. 3f). Olivine is typically altered into serpentine minerals in the matrix, which is subhedral in shape and $40 \mu \mathrm{m}$ in size. Hydrous silicates are amphibole and chlorite. Chlorite is found in the matrix of chromitite (Fig. 3g) while amphibole occurs in chromite grains as inclusions (Fig. 3f, h). These are largely anhedral to subhedral in shape and range in size up to $20 \mu \mathrm{m}$. Occasionally minute sulfide grains occur between chromite grains in the matrix (Fig. 3i).

\section{Analytical Methods}

Petrographic analysis of host rocks and chromitites were carried out with transmitted and reflected light microscopy. Micro X-ray Fluorescence (micro-XRF) was used to analyze the microstructure as well as phase composition. Polished blocks of representative samples were used for the analysis. The X-ray fluorescence analysis was conducted with the Bruker M4 Tornado X-ray apparatus at the Institute of Geoscience at Aarhus University, Denmark. The source used was a single rhodium target X-ray tube. For all samples the following criteria were the same. The spot size used was $20 \mu \mathrm{m}$ and the distance between measured points was $25 \mu \mathrm{m}$. Two spectrometers were running at a range of $40 \mathrm{keV} / 130 \mathrm{kcps}$. The X-ray tube was running at $50 \mathrm{kV}$ electron voltage and the current was set to $600 \mu \mathrm{A}$. All analysis was carried out in vacuum.

The major element composition of chromites and silicate inclusions was analyzed using a JEOL JXA-8600 superprobe at the Department of Geoscience, Aarhus University, Denmark, The method for quantitative analysis was EDS (energy dispersive X-ray spectroscopy) with an acceleration voltage set to $15 \mathrm{KeV}$ and a current beam of $10 \mathrm{nA}$. The beam diameter was $5 \mu \mathrm{m}$ with a counting time of 20 seconds. For the calibration of the microprobe natural mineral standards, including quartz, sanidine, apatite, jadeite, corundum, hematite, rutile, chromite, or oxides $(\mathrm{NiO}, \mathrm{MgO})$ were used. The analysis quality was monitored with two in-house standards; clinopyroxene BB1 and labradorite (Oslo plagioclase). These standards were measured intermittently and compared with the known chemical composition to check the quality of the analysis. They contain all the major elements measured and the relative reproducibility was better than $1 \%$.

The minor and trace element concentrations of chromite were analyzed by LA-ICP-MS at the Aarhus Geochemistry and Isotope Research (AGIR) platform, Department of Geoscience at Aarhus University. The instrument consists of a $193 \mathrm{~nm}$ ArF Excimer laser from Resonetics attached to an Agilent 7900 ICPMS. The analysis was carried out with single spot ablations which had a spot size of $80 \mu \mathrm{m}$. The ablation rate was $8 \mathrm{~Hz}$ at an energy of $80 \mathrm{~mJ} / \mathrm{cm}^{2}$. Helium was used as the carrier gas, which then was mixed with Ar before it entered the ICP. The measurements included 15 seconds of background and 25 seconds of ablation. The NIST 610 reference standards was used as the external calibration standards, and Fe-values determined by electron microprobe/ stoichiometry were used as internal standards. The data processing and quantification was done in Iolite software (Paton, 2011) and follows the procedure outlined in Heinrich et al. (2003).

\section{Results}

\section{Major Element Geochemistry of Chromite}

The composition of fresh chromite grains from the Zhob valley chromitite deposits of all three bodies are given in Table 1. Chromite in these bodies shows a wide range of composition. The range of major element concentration ( $\mathrm{wt} \%$ ) in the chromite from the Zhob body is; $\mathrm{Cr}_{2} \mathrm{O}_{3}$ (39.8-44.9), $\mathrm{Al}_{2} \mathrm{O}_{3}$ (21.3-23.7), $\mathrm{FeO}$ (11.00-13.20), $\mathrm{MgO}$ (12.3417.70) and $\mathrm{TiO}_{2}(0.1-0.3)$. In the Muslim Bagh chromite the values are as $\mathrm{wt} \%$ : $\mathrm{Cr}_{2} \mathrm{O}_{3}(49.4-60.6), \mathrm{Al}_{2} \mathrm{O}_{3}(9.4-17.3), \mathrm{FeO}(10.40-17.50)$, $\mathrm{MgO}(10.30-15.60)$ and $\mathrm{TiO}_{2}(0.01-0.2)$. The chromite from the Khanozai chromitite contains $\mathrm{Cr}_{2} \mathrm{O}_{3}(52.7-55.2), \mathrm{Al}_{2} \mathrm{O}_{3}(9.4-12.9), \mathrm{FeO}$ (11.90-16.30), $\mathrm{MgO}$ (10.30-15.20) and $\mathrm{TiO}_{2}(0.08-0.4)$ (wt\%). Generally, Muslim Bagh and Khanozai chromites have similar composition while the Zhob chromite shows higher $\mathrm{Al}_{2} \mathrm{O}_{3}$ and $\mathrm{MgO}$ values, but lower $\mathrm{Cr}_{2} \mathrm{O}_{3}$ compared to the others (Fig. 4). Nevertheless, nearly all data from all the three chromitite ore deposits fall into the podiform chromitites region on the $\mathrm{TiO}_{2} \mathrm{vs}_{\mathrm{Cr}_{2}} \mathrm{O}_{3}$ diagram (Fig. 5a). The $\mathrm{Al}_{2} \mathrm{O}_{3}$ and $\mathrm{Cr}_{2} \mathrm{O}_{3}$ show a negative correlation (Fig. 5b) in the Zhob 

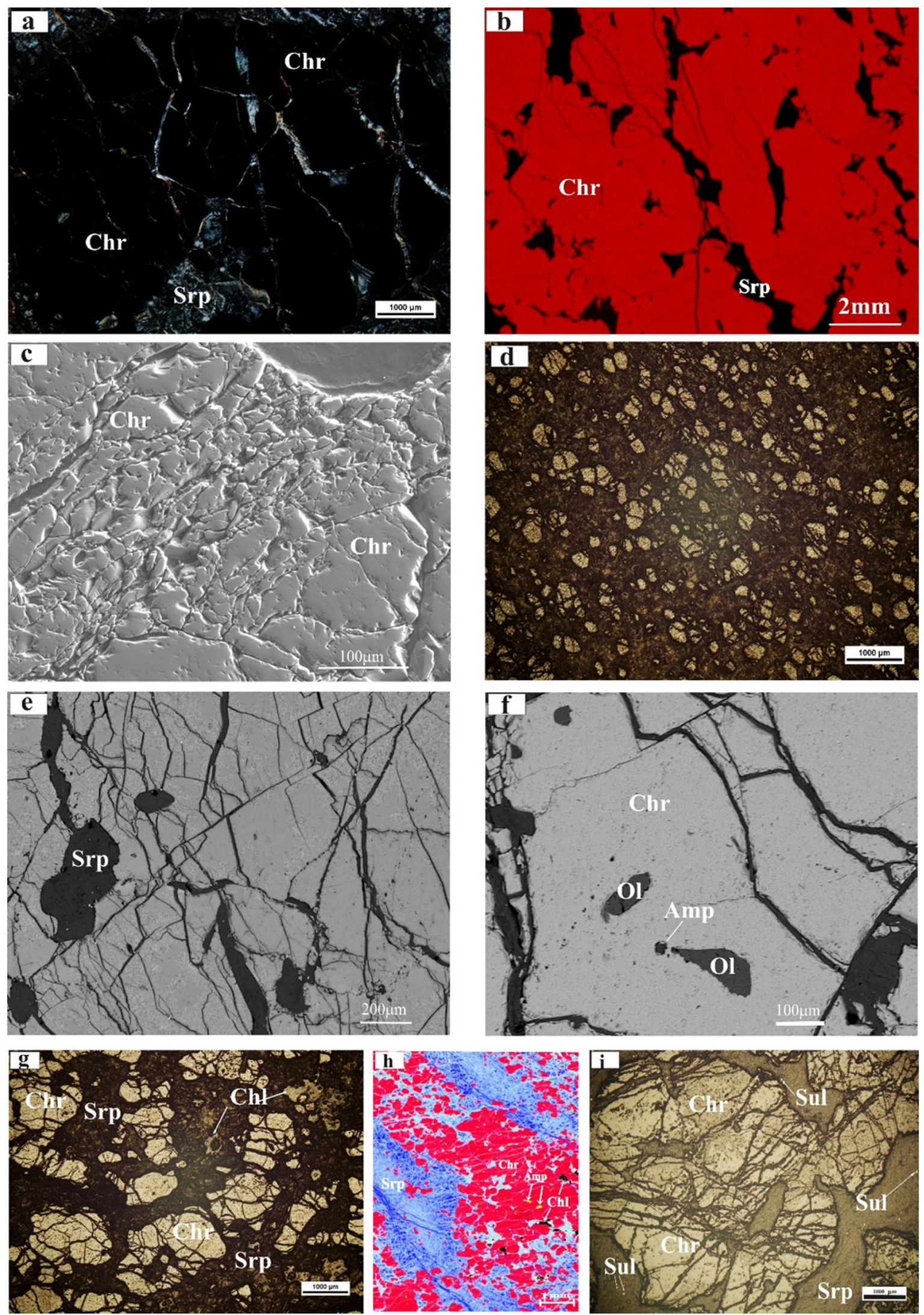

Figure 3. Photomicrographs, micro-XRF, and BSE images showing petrographic characteristics, a) and b) massive-semi massive chromite grains are surrounded by serpentine, $c$ ) chromitites with brecciated texture, d) disseminated texture with subhedral to anhedral grains, e) ferrian alterations of chromitites, $f$ ) olivine and amphibole inclusions in chromite grain, g) chlorite in matrix h) amphibole inclusions in chromite and chlorite in interstitial matrix and i) sulfide grains. Ol=Olivine, Srp=serpentine, Chr=chromite, Amp=Amphibole, Sul=Sulfide. Note that (b) and (h) are micro-XRF images. 
Table 1. Representative chromite compositions (wt\%) of chromitites from the Zhob valley ophiolite and calculated melt compositions. Cr\# = Cr/ $(\mathrm{Cr}+\mathrm{Al}), \mathrm{Mg} \#=\mathrm{Mg} /\left(\mathrm{Mg}+\mathrm{Fe}^{2+}\right), \mathrm{Fe}^{2+} \#=\mathrm{Fe}^{2+} /\left(\mathrm{Mg}+\mathrm{Fe}^{2+}\right)$

\begin{tabular}{|c|c|c|c|c|c|c|c|c|c|c|c|}
\hline \multirow{2}{*}{$\begin{array}{c}\text { Locations } \\
\text { Sample No } \\
\end{array}$} & \multicolumn{11}{|c|}{ Muslim Bagh } \\
\hline & B43 & B43a & B44 & $\mathrm{B} 45 \mathrm{cb}$ & B48 & B49 & B54 & B63 & B64 & B65 & B69 \\
\hline Type & High-Cr & High-Cr & High-Cr & High-Cr & High-Cr & High-Cr & High-Cr & High-Cr & High-Cr & High-Cr & High-Cr \\
\hline $\mathrm{SiO}_{2}$ & 1.50 & 1.30 & 0.10 & 0.20 & 0.50 & 0.40 & 0.00 & 0.20 & 0.10 & 0.80 & 0.40 \\
\hline $\mathrm{TiO} 2$ & 0.19 & 0.10 & 0.14 & 0.08 & 0.19 & 0.20 & 0.10 & 0.06 & 0.01 & 0.20 & 0.10 \\
\hline $\mathrm{Al}_{2} \mathrm{O}_{3}$ & 10.20 & 10.58 & 11.80 & 11.90 & 17.10 & 16.20 & 9.00 & 7.20 & 9.40 & 12.20 & 17.30 \\
\hline $\mathrm{Cr}_{2} \mathrm{O}_{3}$ & 56.50 & 59.58 & 52.70 & 52.90 & 51.20 & 52.10 & 60.00 & 60.60 & 58.10 & 49.40 & 50.80 \\
\hline $\mathrm{Fe}_{2} \mathrm{O}_{3}$ & 2.60 & 0.30 & 6.40 & 6.70 & 3.20 & 3.60 & 3.70 & 5.70 & 4.90 & 7.50 & 4.40 \\
\hline $\mathrm{FeO}$ & 15.30 & 14.80 & 14.80 & 14.70 & 12.60 & 12.90 & 13.20 & 10.40 & 10.40 & 17.50 & 10.90 \\
\hline $\mathrm{MnO}$ & 0.23 & 0.60 & 3.00 & 3.90 & 0.18 & 0.50 & 0.60 & 2.70 & 1.90 & 0.29 & 0.60 \\
\hline $\mathrm{MgO}$ & 13.20 & 11.87 & 10.60 & 10.30 & 15.10 & 14.90 & 12.50 & 13.30 & 13.60 & 11.10 & 15.60 \\
\hline $\mathrm{NiO}$ & 0.10 & 0.10 & 0.20 & 0.20 & 0.21 & 0.10 & 0.40 & 0.20 & 0.10 & 0.10 & 0.00 \\
\hline Total $\Sigma$ & 99.82 & 99.24 & 99.74 & 100.88 & 100.28 & 100.90 & 99.50 & 100.36 & 98.51 & 99.09 & 100.10 \\
\hline \multicolumn{12}{|c|}{ Cations (structural formula on the basis of 4 oxygen) } \\
\hline $\mathrm{Si}$ & 0.04 & 0.00 & 0.00 & 0.00 & 0.01 & 0.01 & 0.00 & 0.00 & 0.00 & 0.02 & 0.01 \\
\hline $\mathrm{Ti}$ & 0.00 & 0.00 & 0.00 & 0.00 & 0.00 & 0.00 & 0.00 & 0.00 & 0.00 & 0.00 & 0.00 \\
\hline $\mathrm{Al}$ & 0.38 & 0.41 & 0.40 & 0.45 & 0.62 & 0.57 & 0.35 & 0.27 & 0.36 & 0.47 & 0.62 \\
\hline $\mathrm{Cr}$ & 1.44 & 1.55 & 1.36 & 1.36 & 1.24 & 1.25 & 1.54 & 1.56 & 1.50 & 1.25 & 1.24 \\
\hline $\mathrm{Fe}^{3+}$ & 0.06 & 0.00 & 0.16 & 0.16 & 0.07 & 0.13 & 0.09 & 0.14 & 0.12 & 0.18 & 0.10 \\
\hline $\mathrm{Fe}^{2+}$ & 0.41 & 0.40 & 0.40 & 0.40 & 0.32 & 0.28 & 0.36 & 0.28 & 0.28 & 0.48 & 0.28 \\
\hline $\mathrm{Mn}$ & 0.00 & 0.00 & 0.08 & 0.10 & 0.00 & 0.01 & 0.01 & 0.07 & 0.05 & 0.00 & 0.00 \\
\hline $\mathrm{Mg}$ & 0.63 & 0.58 & 0.51 & 0.49 & 0.68 & 0.71 & 0.61 & 0.64 & 0.66 & 0.54 & 0.71 \\
\hline $\mathrm{Ni}$ & 0.00 & 0.00 & 0.00 & 0.00 & 0.00 & 0.00 & 0.01 & 0.00 & 0.00 & 0.00 & 0.00 \\
\hline $\mathrm{Cr} \#$ & 0.79 & 0.79 & 0.77 & 0.75 & 0.66 & 0.68 & 0.81 & 0.85 & 0.80 & 0.72 & 0.66 \\
\hline Mg\# & 0.60 & 0.59 & 0.56 & 0.55 & 0.68 & 0.71 & 0.62 & 0.69 & 0.70 & 0.52 & 0.71 \\
\hline $\mathrm{Fe}^{2+} \#$ & 0.39 & 0.40 & 0.43 & 0.44 & 0.32 & 0.28 & 0.37 & 0.30 & 0.29 & 0.47 & 0.28 \\
\hline $\mathrm{Al}_{2} \mathrm{O}_{3}$-melt & 11.06 & 11.25 & 11.82 & 11.87 & 13.76 & 13.48 & 10.41 & 9.25 & 10.64 & 12.00 & 13.82 \\
\hline $\mathrm{TiO}_{2}$-melt & 0.29 & 0.17 & 0.23 & 0.15 & 0.29 & 0.30 & 0.17 & 0.12 & 0.02 & 0.30 & 0.17 \\
\hline Locations & \multicolumn{6}{|c|}{ Khanozai } & & & Zhob & & \\
\hline Sample No & KO30 & KO30a & KO31 & KO32 & KO33 & $\mathrm{KO} 34 \mathrm{~b}$ & Z103c & Z104 & Z105a & Z100 & Z100d \\
\hline Type & High-Cr & High-Cr & High-Cr & High-Cr & High-Cr & High-Cr & High-AL & High-AL & High-AL & High-AL & High-AL \\
\hline $\mathrm{SiO}_{2}$ & 0.10 & 0.10 & 0.50 & 0.60 & 0.50 & 0.30 & 2.10 & 1.80 & 0.40 & 0.19 & 0.16 \\
\hline $\mathrm{TiO}_{2}$ & 0.14 & 0.08 & 0.11 & 0.20 & 0.15 & 0.40 & 0.18 & 0.10 & 0.30 & 0.17 & 0.10 \\
\hline $\mathrm{Al}_{2} \mathrm{O}_{3}$ & 12.80 & 12.90 & 16.20 & 15.20 & 9.40 & 10.30 & 21.90 & 23.70 & 23.20 & 21.30 & 23.23 \\
\hline $\mathrm{Cr}_{2} \mathrm{O}_{3}$ & 52.70 & 52.90 & 51.70 & 53.40 & 55.20 & 54.90 & 42.10 & 39.80 & 44.30 & 44.95 & 42.44 \\
\hline $\mathrm{Fe}_{2} \mathrm{O}_{3}$ & 5.00 & 4.90 & 4.20 & 3.50 & 7.40 & 5.40 & 4.70 & 4.60 & 2.90 & 5.30 & 5.50 \\
\hline $\mathrm{FeO}$ & 15.60 & 16.30 & 11.90 & 12.80 & 14.20 & 16.30 & 11.00 & 12.80 & 13.20 & 12.30 & 11.50 \\
\hline $\mathrm{MnO}$ & 2.00 & 2.00 & 0.16 & 0.50 & 0.23 & 0.80 & 0.20 & 0.40 & 0.30 & 3.28 & 2.46 \\
\hline $\mathrm{MgO}$ & 10.70 & 10.30 & 15.20 & 14.60 & 12.40 & 11.20 & 17.70 & 16.90 & 14.80 & 12.34 & 14.31 \\
\hline $\mathrm{NiO}$ & 0.20 & 0.20 & 0.08 & 0.00 & 0.08 & 0.30 & 0.17 & 0.10 & 0.20 & 0.24 & 0.35 \\
\hline Total $\Sigma$ & 99.24 & 99.68 & 100.05 & 100.80 & 99.56 & 99.90 & 100.05 & 100.20 & 99.60 & 100.07 & 100.05 \\
\hline \multicolumn{12}{|c|}{ Cations (structural formula on the basis of 4 oxygen) } \\
\hline $\mathrm{Si}$ & 0.00 & 0.00 & 0.01 & 0.10 & 0.00 & 0.00 & 0.06 & 0.05 & 0.01 & 0.00 & 0.00 \\
\hline $\mathrm{Ti}$ & 0.00 & 0.00 & 0.00 & 0.00 & 0.00 & 0.00 & 0.00 & 0.00 & 0.00 & 0.00 & 0.00 \\
\hline $\mathrm{Al}$ & 0.49 & 0.49 & 0.59 & 0.55 & 0.36 & 0.39 & 0.76 & 0.83 & 0.83 & 0.76 & 0.83 \\
\hline $\mathrm{Cr}$ & 1.36 & 1.36 & 1.27 & 1.30 & 1.43 & 1.42 & 0.99 & 0.94 & 1.06 & 1.08 & 1.02 \\
\hline $\mathrm{Fe}^{3+}$ & 0.12 & 0.12 & 0.09 & 0.08 & 0.18 & 0.13 & 0.10 & 0.10 & 0.06 & 0.17 & 0.12 \\
\hline $\mathrm{Fe}^{2+}$ & 0.42 & 0.44 & 0.30 & 0.33 & 0.38 & 0.44 & 0.27 & 0.32 & 0.33 & 0.33 & 0.29 \\
\hline $\mathrm{Mn}$ & 0.05 & 0.05 & 0.00 & 0.10 & 0.00 & 0.02 & 0.00 & 0.02 & 0.00 & 0.08 & 0.06 \\
\hline $\mathrm{Mg}$ & 0.52 & 0.50 & 0.70 & 0.67 & 0.60 & 0.54 & 0.78 & 0.71 & 0.67 & 0.60 & 0.64 \\
\hline $\mathrm{Ni}$ & 0.00 & 0.00 & 0.00 & 0.00 & 0.00 & 0.00 & 0.00 & 0.00 & 0.00 & 0.00 & 0.00 \\
\hline $\mathrm{Cr} \#$ & 0.73 & 0.73 & 0.68 & 0.70 & 0.79 & 0.78 & 0.56 & 0.53 & 0.56 & 0.58 & 0.55 \\
\hline Mg\# & 0.55 & 0.53 & 0.70 & 0.67 & 0.61 & 0.55 & 0.74 & 0.68 & 0.67 & 0.64 & 0.68 \\
\hline $\mathrm{Fe}^{2+} \#$ & 0.44 & 0.46 & 0.30 & 0.33 & 0.38 & 0.44 & 0.25 & 0.31 & 0.33 & 0.35 & 0.31 \\
\hline $\mathrm{Al}_{2} \mathrm{O}_{3}$-melt & 12.25 & 12.29 & 13.48 & 13.14 & 10.64 & 11.11 & 15.05 & 15.46 & 15.35 & 14.91 & 15.36 \\
\hline $\mathrm{TiO}_{2}$-melt & 0.23 & 0.15 & 0.19 & 0.30 & 0.24 & 0.53 & 0.28 & 0.17 & 0.42 & 0.27 & 0.17 \\
\hline
\end{tabular}




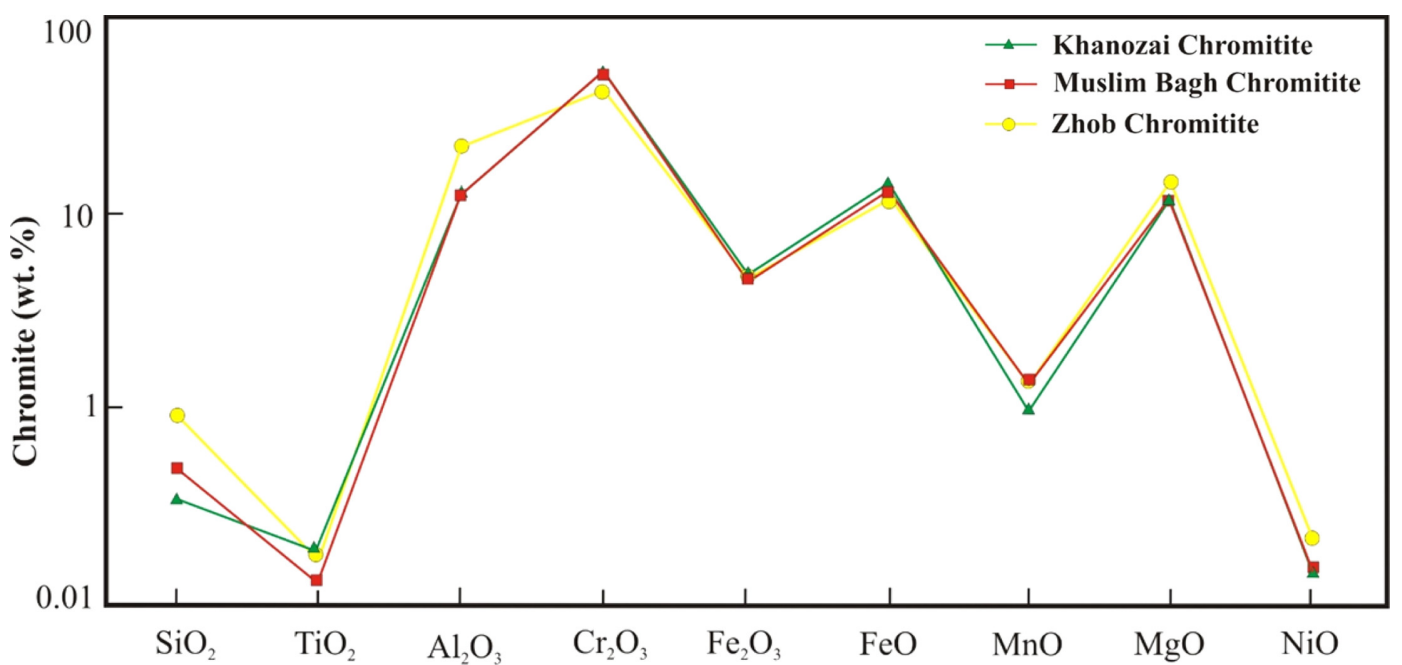

Figure 4. Comparison of major elements in the three chromite occurrences. Average concentrations of major elements in chromites from Khanozai, Muslim Bagh and Zhob ore deposits, showing uniform compositions with slight differences in $\mathrm{SiO}_{2}, \mathrm{Al}_{2} \mathrm{O}_{3}$ and $\mathrm{MnO}$.
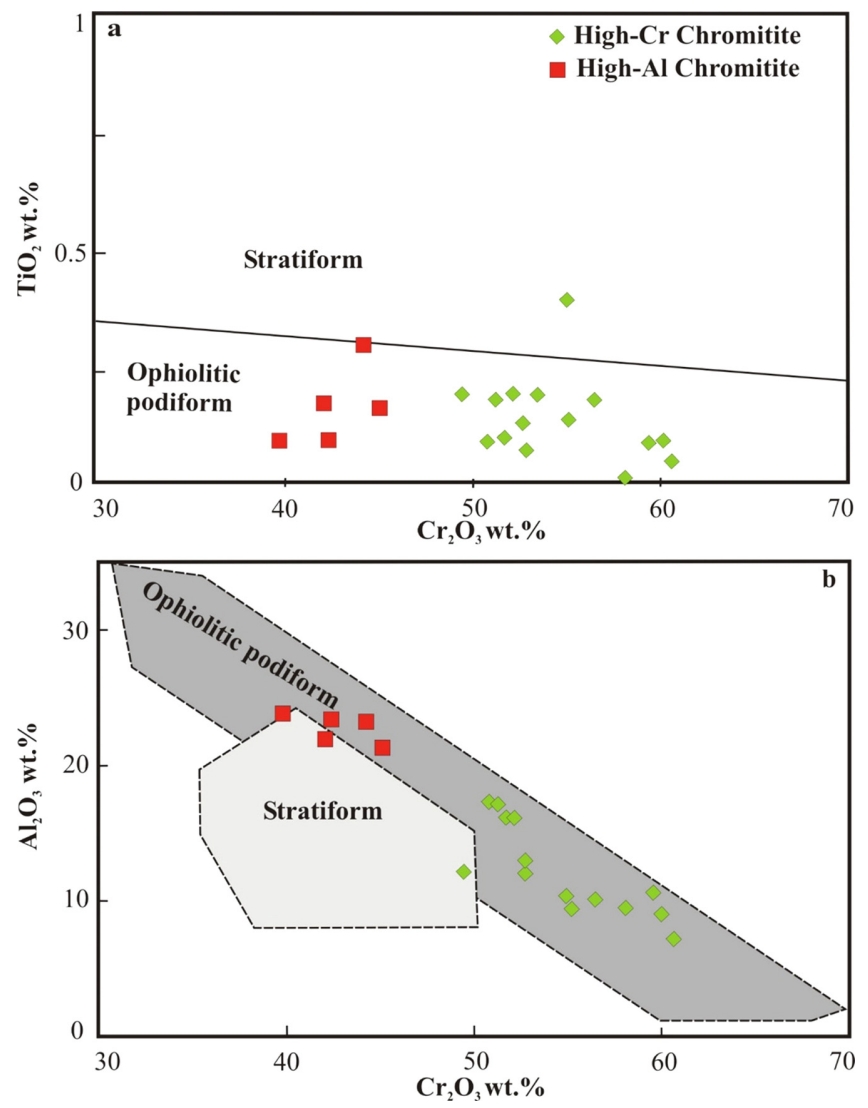

Figure 5. Composition of chromite from Zhob valley chromitites plotted on (a) $\mathrm{TiO}_{2}$ vs $\mathrm{Cr}_{2} \mathrm{O}_{3}$ (b) $\mathrm{Al}_{2} \mathrm{O}_{3}$ and $\mathrm{Cr}_{2} \mathrm{O}_{3}$ diagrams. Fields of podiform and stratifom chromitites are from Bonovia et al., (1993) and Ahmed and Arai (2003).

valley chromitites which is characteristic for ophiolitic chromitites.

On the basis of compositional variation of chromite, the studied chromitites can be classified into two groups as high- $\mathrm{Cr}(\mathrm{Cr} \#=\mathrm{Cr} /(\mathrm{Cr}+\mathrm{Al})$ $>0.6)$ and high- $\mathrm{Al}(\mathrm{Cr} \#=\mathrm{Cr} /(\mathrm{Cr}+\mathrm{Al})<0.6)$. Muslim Bagh and Khanozai chromitites are high-Cr composition which contains high $\mathrm{Cr} \#$ values ranging from 0.66 to 0.85 and from 0.73 to 0.79 , respectively. In contrast, the Zhob chromitite is high-Al character and shows $\mathrm{Cr} \#$ values from 0.53 to 0.58 . Generally, both high- $\mathrm{Cr}$ and high- $\mathrm{Al}$ chromitites are low in $\mathrm{Fe}_{2} \mathrm{O}_{3}$ concentrations (approx. $5 \mathrm{wt} \%$ ) but a few samples from high-Cr chromitites contain higher $\mathrm{Fe}_{2} \mathrm{O}_{3}$ contents, reaching up to $7.4 \mathrm{wt} \%$.

Profile analyses of chromite are mostly unzoned but a few grains in high-Cr and high-Al chromite show weak and strong zonation, respectively. The zoned chromite grains from high-Al chromitite exhibit an increase from rim to core in $\mathrm{Mg} \#$ (Fig. 6a). But $\mathrm{Cr} \#$ and $\mathrm{FeO}_{\text {total }}$ (wt\%) values decrease from rim to core in grains from high-Al chromitite (Fig. 6a). On other hand, values of $\mathrm{Cr} \#, \mathrm{Mg} \#$ and $\mathrm{FeO}_{\text {total }}(\mathrm{wt} \%$ ) are approximately uniform from rim to core in chromite grains from high$\mathrm{Cr}$ chromitites but some grains show slight zonation. In high-Cr chromitites, values of $\mathrm{Cr} \#$ increase from rim to core while $\mathrm{FeO}_{\text {total }}(\mathrm{wt} \%)$ values decrease from rim to core and $\mathrm{Mg} \#$ values increase partially from rim to core (Fig. 6b).

\section{Minor and Trace Element Geochemistry of Chromite}

The 174 spots on fresh parts of chromite grains were analysed for minor and trace element concentrations $(\mathrm{Sc}, \mathrm{Ti}, \mathrm{V}, \mathrm{Mn}, \mathrm{Co}, \mathrm{Ni}, \mathrm{Cu}$, $\mathrm{Zn}$, and $\mathrm{Ga}$ ) in both high-Cr and high-Al chromitites. The average contents are listed in Table 2. Average contents of Ti, V, Ni, Zn and $\mathrm{Ga}$ in chromite of high-Cr chromitites $(1523,718,1002,551$, and $26.2 \mathrm{ppm}$, respectively) are lower than those in high-Al chromitites (1880, 1158, 1979, 730.5, and 64.3 ppm, respectively) (Fig. 7). Average concentrations of $\mathrm{Sc}$ and $\mathrm{Mn}$ are higher in chromite ( 9 and $2264 \mathrm{ppm}$, respectively) of high-Cr chromitites than those in the high-Al chromitites (5.9 and $1882 \mathrm{ppm}$, respectively) while average contents of $\mathrm{Co}$ and $\mathrm{Cu}$ are approximately similar in both types of chromitites (Fig. 7).

\section{Silicate Inclusions}

Olivine and amphibole are the primary silicate inclusions found in fresh grains of chromite. In both types of chromitites, the chemical 

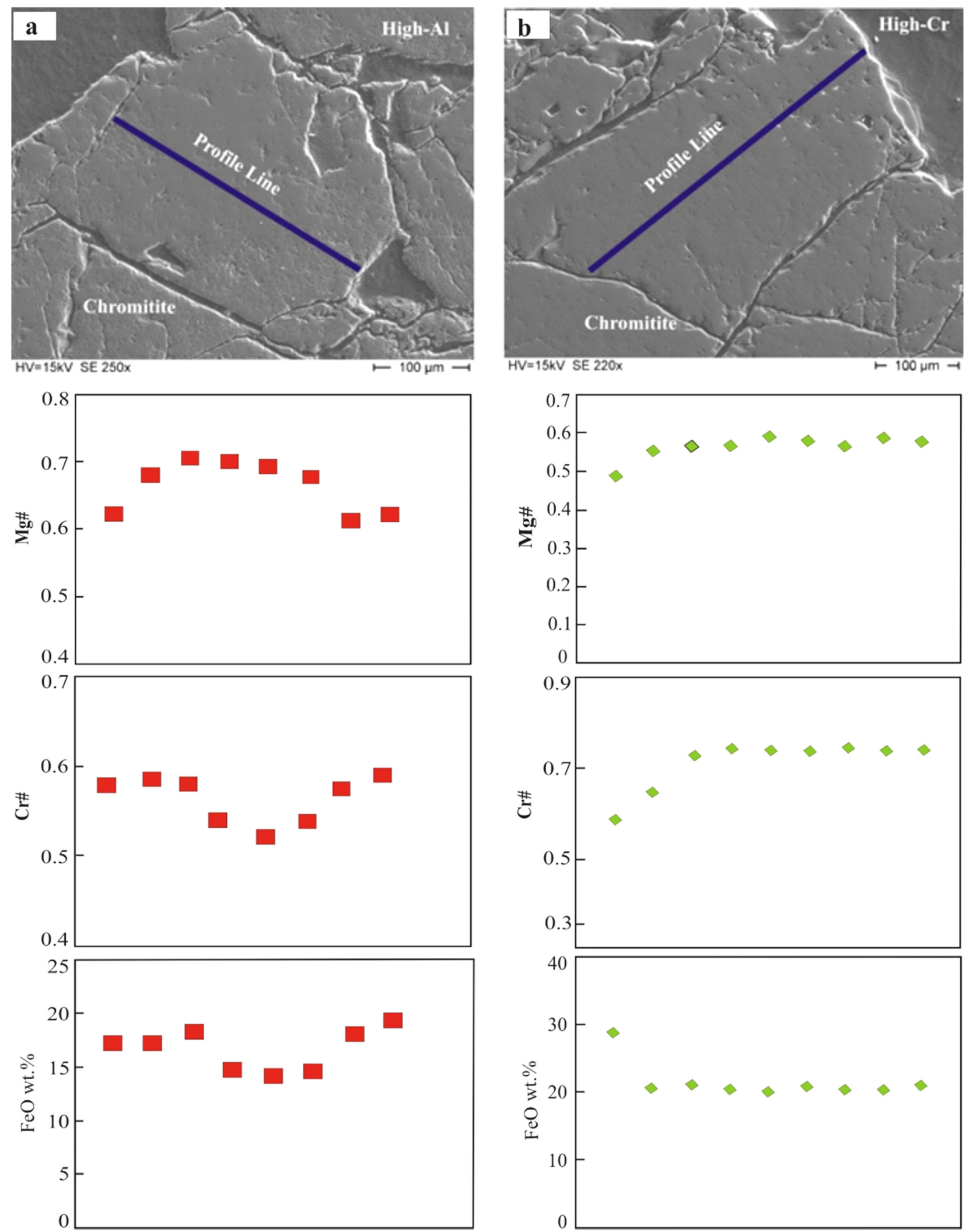

Figure 6. Profile analysis of chromite grains from Zhob valley chromitites; (a) high-Al and (b) high-Cr chromitite.

composition of these inclusions are almost similar (Table 3). Olivine is the most common inclusions that contains up to $0.80 \mathrm{wt} \% \mathrm{NiO}$ and $0.5 \mathrm{wt} \% \mathrm{MnO}$ which is represented by high Fo content (average $\mathrm{Mg \#}$
$=0.97$, Table 3), showing forsterite composition. Amphibole contains high $\mathrm{Mg} \#$ ranges from 0.92 to 0.96 and $\mathrm{Si}$ contents ranges from 6.53 to 7.25 which classify amphibole as magnesio-hornblende. Major ele- 
Table 2. Minor and trace elements concentrations (ppm) of representative chromites from the chromitites of Zhob valley ophiolite

\begin{tabular}{|c|c|c|c|c|c|c|c|c|c|c|}
\hline Element & & Sc & $\overline{\mathrm{Ti}}$ & $\mathrm{V}$ & $\mathrm{Mn}$ & $\mathrm{Co}$ & $\mathrm{Ni}$ & $\mathrm{Cu}$ & $\mathrm{Zn}$ & $\mathrm{Ga}$ \\
\hline Muslim Bagh & Type & & & & & & & & & \\
\hline B43-1 & High-Cr & 7.52 & 1752 & 569 & 2161 & 342 & 1177 & 1.49 & 578 & 30.20 \\
\hline B43-2 & High-Cr & 8.87 & 1726 & 558 & 2391 & 367 & 962 & 2.87 & 636 & 28.70 \\
\hline B43-3 & High-Cr & 8.99 & 1745 & 563 & 2401 & 369 & 988 & 4.80 & 620 & 28.60 \\
\hline B43-4 & High-Cr & 7.83 & 1743 & 572 & 2250 & 359 & 1112 & 2.00 & 574 & 29.10 \\
\hline B43-5 & High-Cr & 8.58 & 1780 & 578 & 2397 & 373 & 1024 & 2.33 & 613 & 31.00 \\
\hline B43-6 & High-Cr & 7.59 & 1743 & 575 & 2183 & 350 & 1168 & 1.54 & 589 & 30.30 \\
\hline B43-7 & High-Cr & 7.90 & 1741 & 583 & 2226 & 352 & 1190 & 1.21 & 583 & 30.90 \\
\hline B43-8 & High-Cr & 7.99 & 1789 & 577 & 2065 & 335 & 1285 & 1.53 & 569 & 31.08 \\
\hline B43-9 & High-Cr & 8.71 & 1804 & 588 & 2350 & 375 & 1032 & 1.27 & 585 & 31.90 \\
\hline B43-10 & High-Cr & 9.00 & 1762 & 580 & 2437 & 371 & 973 & 0.54 & 617 & 31.10 \\
\hline B43a-1 & High-Cr & 7.71 & 1741 & 561 & 2063 & 340 & 1186 & 0.38 & 581 & 30.10 \\
\hline B43a-2 & High-Cr & 7.57 & 1774 & 577 & 2076 & 343 & 1221 & 1.76 & 576 & 30.44 \\
\hline B43a-3 & High-Cr & 7.89 & 1776 & 580 & 2272 & 363 & 1179 & 0.89 & 593 & 30.58 \\
\hline B43a-4 & High-Cr & 7.88 & 1774 & 580 & 2332 & 371 & 1033 & 1.17 & 587 & 31.60 \\
\hline B43a-5 & High-Cr & 8.28 & 1732 & 573 & 2281 & 369 & 1092 & 1.33 & 586 & 30.30 \\
\hline B43a-6 & High-Cr & 8.04 & 1749 & 581 & 2145 & 349 & 1232 & 1.79 & 578 & 31.70 \\
\hline B43a-7 & High-Cr & 7.46 & 1768 & 576 & 2136 & 352 & 1239 & 2.12 & 583 & 30.50 \\
\hline B43a-8 & High-Cr & 7.70 & 1716 & 575 & 2191 & 342 & 1237 & 3.20 & 613 & 30.30 \\
\hline B43a-9 & High-Cr & 7.38 & 1761 & 573 & 2121 & 342 & 1211 & 1.36 & 559 & 30.10 \\
\hline B43a-10 & High-Cr & 8.16 & 1747 & 569 & 2172 & 349 & 1185 & 1.65 & 610 & 30.80 \\
\hline B43a-11 & High-Cr & 8.23 & 1783 & 583 & 2383 & 383 & 952 & 0.83 & 617 & 29.00 \\
\hline B43a-12 & High-Cr & 9.18 & 1763 & 577 & 2402 & 385 & 949 & 0.90 & 603 & 28.30 \\
\hline B43a-13 & High-Cr & 7.90 & 1789 & 580 & 2030 & 334 & 1265 & 2.19 & 586 & 30.70 \\
\hline B43a-14 & High-Cr & 7.92 & 1738 & 574 & 2233 & 356 & 1118 & 0.71 & 579 & 30.00 \\
\hline B43a-15 & High-Cr & 7.47 & 1755 & 580 & 2083 & 341 & 1241 & 5.00 & 574 & 30.60 \\
\hline B43a-16 & High-Cr & 6.85 & 1744 & 576 & 2059 & 334 & 1253 & 0.65 & 583 & 30.03 \\
\hline B43a-17 & High-Cr & 7.73 & 1711 & 567 & 2124 & 333 & 1246 & 2.78 & 557 & 31.00 \\
\hline B43a-18 & High-Cr & 7.81 & 1746 & 572 & 2040 & 334 & 1256 & 1.15 & 563 & 31.60 \\
\hline B43a-19 & High-Cr & 7.90 & 1747 & 574 & 2037 & 335 & 1254 & 1.16 & 572 & 29.60 \\
\hline B43a-20 & High-Cr & 8.30 & 1749 & 577 & 2135 & 345 & 1194 & 16.00 & 568 & 30.10 \\
\hline B43a-21 & High-Cr & 8.49 & 1786 & 574 & 2406 & 378 & 966 & 0.45 & 632 & 29.30 \\
\hline B43a-22 & High-Cr & 7.66 & 1738 & 569 & 2388 & 372 & 942 & 0.82 & 593 & 28.90 \\
\hline ST DEV & & 0.53 & 22.11 & 6 & 135 & 16 & 115.49 & 2.76 & 20.71 & 0.94 \\
\hline Average & & 8.01 & 1755 & 575 & 2218 & 354 & 1136.3 & 2.12 & 589.3 & 30.26 \\
\hline B65-1 & High-Cr & 11.67 & 1876 & 877 & 2894 & 359 & 1051 & 1.34 & 730 & 32.80 \\
\hline B65-2 & High-Cr & 11.03 & 1920 & 888 & 2892 & 357 & 1061 & 1.01 & 701 & 33.70 \\
\hline B65-3 & High-Cr & 12.32 & 1929 & 895 & 2937 & 377 & 1010 & 13.00 & 694 & 34.00 \\
\hline B65-4 & High-Cr & 14.20 & 1880 & 884 & 3555 & 395 & 3040 & 3.00 & 816 & 35.80 \\
\hline B65-5 & High-Cr & 10.89 & 1931 & 898 & 2938 & 377 & 1063 & 0.98 & 704 & 33.60 \\
\hline B65-6 & High-Cr & 10.99 & 1882 & 879 & 2956 & 377 & 1021 & 0.78 & 703 & 34.00 \\
\hline B65-7 & High-Cr & 11.21 & 1870 & 879 & 2895 & 365 & 1055 & 0.74 & 668 & 32.00 \\
\hline B65-8 & High-Cr & 11.53 & 1953 & 912 & 2911 & 367 & 1078 & 0.61 & 688 & 33.30 \\
\hline B65-9 & High-Cr & 11.47 & 1993 & 937 & 2941 & 372 & 1068 & 0.57 & 699 & 35.20 \\
\hline B65-10 & High-Cr & 11.51 & 1926 & 901 & 2916 & 359 & 1058 & 2.30 & 700 & 33.70 \\
\hline B65-11 & High-Cr & 10.57 & 1887 & 907 & 2852 & 358 & 1088 & 1.65 & 700 & 34.00 \\
\hline B69-1 & High-Cr & 10.83 & 1852 & 888 & 2917 & 363 & 1033 & 1.25 & 701 & 33.60 \\
\hline B69-2 & High-Cr & 11.82 & 1890 & 904 & 2916 & 362 & 1046 & 1.31 & 699 & 34.40 \\
\hline
\end{tabular}


Table 2. (Continued)

\begin{tabular}{|c|c|c|c|c|c|c|c|c|c|c|}
\hline Element & & $\overline{\mathrm{Sc}}$ & $\overline{\mathrm{TT}}$ & $\overline{\mathrm{V}}$ & $\mathrm{Mn}$ & $\overline{\mathrm{CO}}$ & $\mathrm{Ni}$ & $\overline{\mathrm{Cu}}$ & $\overline{\mathrm{Zn}}$ & $\overline{\mathrm{Ga}}$ \\
\hline \multicolumn{11}{|c|}{ Type } \\
\hline B69-3 & High-Cr & 11.21 & 1912 & 919 & 2916 & 361 & 1081 & 1.06 & 705 & 35.60 \\
\hline B69-4 & High-Cr & 11.13 & 1942 & 926 & 2835 & 360 & 1124 & 0.77 & 718 & 34.70 \\
\hline B69-5 & High-Cr & 11.82 & 1934 & 920 & 2971 & 379 & 1067 & 0.82 & 706 & 34.10 \\
\hline B69-6 & High-Cr & 11.25 & 1920 & 928 & 2908 & 366 & 1078 & 1.26 & 691 & 34.60 \\
\hline B69-7 & High-Cr & 11.04 & 1988 & 954 & 2954 & 364 & 1062 & 0.78 & 701 & 34.80 \\
\hline B69-8 & High-Cr & 11.89 & 1932 & 944 & 2888 & 365 & 1124 & 1.08 & 717 & 34.60 \\
\hline B69-9 & High-Cr & 11.84 & 1952 & 929 & 2869 & 365 & 1100 & 0.96 & 688 & 35.30 \\
\hline B69-10 & High-Cr & 11.36 & 1921 & 933 & 2884 & 367 & 1043 & 1.24 & 728 & 34.00 \\
\hline B69-11 & High-Cr & 11.68 & 1916 & 924 & 2846 & 357 & 1091 & 1.55 & 699 & 34.00 \\
\hline B69-12 & High-Cr & 11.54 & 1976 & 947 & 2965 & 367 & 1092 & 0.67 & 700 & 33.90 \\
\hline B69-13 & High-Cr & 11.95 & 2006 & 951 & 2954 & 380 & 1098 & 0.81 & 728 & 34.70 \\
\hline B69-14 & High-Cr & 13.10 & 1930 & 940 & 3056 & 385 & 1041 & 0.94 & 724 & 34.80 \\
\hline B69-15 & High-Cr & 12.07 & 2006 & 951 & 2915 & 369 & 1075 & 0.75 & 740 & 34.20 \\
\hline B69-16 & High-Cr & 12.23 & 2019 & 954 & 2963 & 375 & 1052 & 0.67 & 743 & 34.60 \\
\hline B69-17 & High-Cr & 11.71 & 1976 & 948 & 2837 & 363 & 1152 & 0.82 & 738 & 37.00 \\
\hline B69-18 & High-Cr & 11.77 & 2011 & 956 & 2867 & 369 & 1114 & 0.89 & 732 & 35.50 \\
\hline B69-19 & High-Cr & 12.13 & 1977 & 949 & 2976 & 383 & 1044 & 1.13 & 711 & 34.30 \\
\hline ST DEV & & 0.71 & 45.94 & 26 & 126 & 9 & 360.81 & 2.23 & 26.30 & 0.95 \\
\hline Average & & 11.65 & 1937 & 921 & 2937 & 369 & 1137 & 1.49 & 712.4 & 34.36 \\
\hline \multicolumn{11}{|l|}{ Khanozai } \\
\hline KO33-1 & High-Cr & 9.96 & 1506 & 595 & 2271 & 373 & 836 & 1.90 & 508 & 23.99 \\
\hline KO33-2 & High-Cr & 9.75 & 1535 & 597 & 2349 & 374 & 865 & 1.64 & 493 & 25.30 \\
\hline KO33-3 & High-Cr & 9.65 & 1492 & 602 & 2275 & 368 & 873 & 1.80 & 479 & 24.66 \\
\hline KO33-4 & High-Cr & 9.29 & 1476 & 592 & 2305 & 371 & 829 & 1.60 & 492 & 23.60 \\
\hline KO33-5 & High-Cr & 9.16 & 1471 & 597 & 2345 & 371 & 840 & 2.00 & 502 & 24.49 \\
\hline KO33-6 & High-Cr & 9.50 & 1470 & 596 & 2331 & 369 & 843 & 1.57 & 497 & 24.50 \\
\hline KO33-7 & High-Cr & 9.35 & 1471 & 590 & 2252 & 366 & 840 & 1.61 & 493 & 24.40 \\
\hline KO33-8 & High-Cr & 8.28 & 1471 & 594 & 2266 & 365 & 833 & 2.02 & 509 & 24.40 \\
\hline KO33-9 & High-Cr & 9.38 & 1530 & 608 & 2329 & 375 & 853 & 1.85 & 500 & 25.67 \\
\hline KO33-10 & High-Cr & 8.16 & 1498 & 594 & 2260 & 372 & 821 & 1.68 & 498 & 24.18 \\
\hline KO33-11 & High-Cr & 9.11 & 1437 & 585 & 2282 & 365 & 831 & 1.74 & 490 & 24.17 \\
\hline KO33-12 & High-Cr & 9.39 & 1512 & 593 & 2289 & 366 & 837 & 2.10 & 503 & 24.12 \\
\hline KO33-13 & High-Cr & 7.33 & 1494 & 591 & 2334 & 370 & 841 & 1.89 & 495 & 23.87 \\
\hline KO33-14 & High-Cr & 8.40 & 1403 & 573 & 2287 & 365 & 799 & 1.62 & 506 & 23.23 \\
\hline KO33-15 & High-Cr & 9.08 & 1511 & 591 & 2314 & 371 & 836 & 1.84 & 512 & 24.20 \\
\hline KO33-16 & High-Cr & 9.87 & 1472 & 589 & 2336 & 368 & 759 & 1.62 & 519 & 25.00 \\
\hline KO33-17 & High-Cr & 8.04 & 1462 & 574 & 2260 & 371 & 814 & 2.69 & 507 & 23.10 \\
\hline KO33-18 & High-Cr & 9.46 & 1523 & 587 & 2284 & 373 & 846 & 1.86 & 523 & 23.22 \\
\hline KO33-19 & High-Cr & 9.22 & 1512 & 588 & 2316 & 378 & 839 & 1.39 & 514 & 25.00 \\
\hline KO33-20 & High-Cr & 9.73 & 1532 & 594 & 2330 & 376 & 838 & 2.16 & 519 & 24.30 \\
\hline KO33-21 & High-Cr & 7.32 & 1521 & 601 & 2344 & 374 & 840 & 1.55 & 527 & 24.29 \\
\hline KO33-22 & High-Cr & 8.50 & 1557 & 599 & 2378 & 381 & 861 & 2.10 & 527 & 23.86 \\
\hline KO33-23 & High-Cr & 9.73 & 1507 & 587 & 2388 & 378 & 850 & 1.71 & 503 & 23.10 \\
\hline KO33-24 & High-Cr & 8.53 & 1506 & 591 & 2358 & 377 & 846 & 1.77 & 513 & 24.60 \\
\hline KO33-25 & High-Cr & 9.05 & 1503 & 584 & 2376 & 381 & 856 & 1.56 & 530 & 23.51 \\
\hline KO33-26 & High-Cr & 9.55 & 1525 & 580 & 2419 & 391 & 892 & 1.82 & 552 & 23.19 \\
\hline KO33-27 & High-Cr & 8.34 & 1491 & 577 & 2341 & 381 & 844 & 1.66 & 527 & 23.24 \\
\hline
\end{tabular}




\begin{tabular}{|c|c|c|c|c|c|c|c|c|c|c|}
\hline Element & & $\mathrm{Sc}$ & $\overline{\mathrm{Ti}}$ & $\mathrm{V}$ & $\mathrm{Mn}$ & $\overline{\mathrm{Co}}$ & $\mathrm{Ni}$ & $\mathrm{Cu}$ & $\overline{\mathrm{Zn}}$ & $\overline{\mathrm{Ga}}$ \\
\hline \multicolumn{11}{|c|}{ Type } \\
\hline ST DEV & & 0.73 & 32.73 & 8 & 44 & 6 & 24.05 & 0.26 & 15.74 & 0.69 \\
\hline Average & & 9.01 & 1496 & 591 & 2319 & 373 & 839.33 & 1.80 & 508.8 & 24.11 \\
\hline KO31-1 & High-Cr & 6.55 & 1096 & 557 & 1571 & 263 & 887 & 4.94 & 384 & 19.03 \\
\hline KO31-2 & High-Cr & 6.55 & 1126 & 551 & 1586 & 265 & 881 & 6.12 & 388 & 18.96 \\
\hline KO31-3 & High-Cr & 6.34 & 1143 & 547 & 1549 & 261 & 881 & 6.32 & 385 & 18.57 \\
\hline KO31-4 & High-Cr & 6.77 & 1128 & 539 & 1604 & 260 & 835 & 3.54 & 387 & 18.36 \\
\hline KO31-5 & High-Cr & 6.27 & 1125 & 530 & 1552 & 261 & 911 & 8.50 & 384 & 18.44 \\
\hline KO31-6 & High-Cr & 6.49 & 1112 & 511 & 1570 & 263 & 899 & 9.16 & 371 & 18.30 \\
\hline KO31-7 & High-Cr & 6.56 & 1128 & 496 & 1608 & 263 & 873 & 5.33 & 375 & 17.91 \\
\hline KO31-8 & High-Cr & 6.49 & 1111 & 493 & 1594 & 268 & 846 & 4.42 & 388 & 18.01 \\
\hline KO31-9 & High-Cr & 6.08 & 1106 & 476 & 1572 & 263 & 878 & 5.88 & 379 & 18.72 \\
\hline KO31-10 & High-Cr & 6.23 & 1106 & 470 & 1592 & 263 & 876 & 5.48 & 381 & 18.05 \\
\hline KO31-11 & High-Cr & 6.98 & 1109 & 474 & 1585 & 263 & 866 & 5.32 & 372 & 17.98 \\
\hline KO31-12 & High-Cr & 6.43 & 1098 & 468 & 1560 & 261 & 874 & 5.98 & 370 & 17.55 \\
\hline KO31-13 & High-Cr & 6.30 & 1099 & 510 & 1565 & 258 & 881 & 5.31 & 372 & 17.70 \\
\hline KO31-14 & High-Cr & 6.28 & 1125 & 520 & 1550 & 261 & 941 & 8.65 & 375 & 18.70 \\
\hline KO31-15 & High-Cr & 6.80 & 1102 & 522 & 1576 & 263 & 867 & 5.86 & 382 & 18.18 \\
\hline KO31-16 & High-Cr & 6.61 & 1140 & 552 & 1570 & 262 & 892 & 4.00 & 389 & 19.93 \\
\hline KO31-17 & High-Cr & 6.41 & 1114 & 537 & 1538 & 264 & 892 & 6.50 & 379 & 18.54 \\
\hline KO31-18 & High-Cr & 6.32 & 1069 & 508 & 1621 & 268 & 821 & 4.93 & 367 & 18.52 \\
\hline KO31-19 & High-Cr & 6.58 & 1112 & 521 & 1610 & 266 & 860 & 3.34 & 369 & 17.58 \\
\hline KO31-20 & High-Cr & 6.44 & 1123 & 524 & 1582 & 269 & 881 & 5.79 & 374 & 18.90 \\
\hline KO31-21 & High-Cr & 6.48 & 1094 & 496 & 1608 & 265 & 829 & 1.12 & 376 & 16.93 \\
\hline KO31-22 & High-Cr & 6.63 & 1087 & 502 & 1588 & 266 & 841 & 4.92 & 369 & 18.58 \\
\hline KO31-23 & High-Cr & 6.22 & 1098 & 479 & 1594 & 266 & 875 & 5.64 & 381 & 18.10 \\
\hline KO31-24 & High-Cr & 6.57 & 1101 & 477 & 1594 & 267 & 882 & 5.72 & 383 & 18.41 \\
\hline KO31-25 & High-Cr & 6.27 & 1092 & 476 & 1575 & 264 & 857 & 3.80 & 371 & 17.99 \\
\hline KO31-26 & High-Cr & 6.67 & 1018 & 455 & 1626 & 271 & 781 & 3.42 & 379 & 17.04 \\
\hline KO31-27 & High-Cr & 6.46 & 1075 & 466 & 1592 & 263 & 851 & 3.53 & 393 & 18.52 \\
\hline KO31-28 & High-Cr & 6.56 & 1139 & 489 & 1580 & 265 & 877 & 4.92 & 381 & 19.02 \\
\hline KO31-29 & High-Cr & 6.90 & 1100 & 497 & 1572 & 264 & 910 & 5.74 & 368 & 18.42 \\
\hline KO31-30 & High-Cr & 6.74 & 1123 & 500 & 1573 & 268 & 879 & 5.11 & 378 & 17.79 \\
\hline KO31-31 & High-Cr & 6.42 & 1110 & 546 & 1566 & 277 & 858 & 4.39 & 392 & 17.87 \\
\hline KO31-32 & High-Cr & 6.71 & 1127 & 568 & 1584 & 268 & 863 & 4.12 & 385 & 18.41 \\
\hline KO31-33 & High-Cr & 6.02 & 1140 & 566 & 1570 & 267 & 875 & 4.98 & 379 & 18.90 \\
\hline KO31-34 & High-Cr & 6.59 & 1162 & 574 & 1574 & 268 & 876 & 4.66 & 372 & 18.59 \\
\hline KO31-35 & High-Cr & 6.45 & 1116 & 550 & 1565 & 270 & 897 & 7.00 & 382 & 19.29 \\
\hline KO31-36 & High-Cr & 6.36 & 1132 & 556 & 1603 & 270 & 882 & 5.79 & 374 & 17.92 \\
\hline KO31-37 & High-Cr & 6.56 & 1139 & 574 & 1568 & 268 & 894 & 4.11 & 381 & 18.69 \\
\hline KO31-38 & High-Cr & 6.22 & 1057 & 550 & 1580 & 258 & 839 & 3.24 & 352 & 17.25 \\
\hline KO31-39 & High-Cr & 6.18 & 1115 & 524 & 2011 & 347 & 709 & 1.88 & 492 & 16.90 \\
\hline ST DEV & & 0.21 & 16.15 & 34 & 72 & 14 & 38.01 & 1.61 & 19.96 & 0.64 \\
\hline Average & & 6.47 & 1110 & 517 & 1592 & 267 & 867.1 & 5.11 & 381 & 18.27 \\
\hline \multicolumn{11}{|l|}{ Zhob } \\
\hline Z100-1 & High-Al & 4.92 & 1845 & 1339 & 1894 & 307 & 1786 & 0.00 & 880 & 72.90 \\
\hline Z100-2 & High-Al & 4.32 & 1919 & 1347 & 1734 & 297 & 2008 & 1.27 & 788 & 70.90 \\
\hline Z100-3 & High-Al & 5.22 & 1871 & 1282 & 1786 & 289 & 1765 & 2.60 & 784 & 62.90 \\
\hline
\end{tabular}


Table 2. (Continued)

\begin{tabular}{|c|c|c|c|c|c|c|c|c|c|c|}
\hline Element & & $\mathrm{Sc}$ & $\mathrm{Ti}$ & $\mathrm{V}$ & Mn & $\mathrm{Co}$ & $\mathrm{Ni}$ & $\mathrm{Cu}$ & $\mathrm{Zn}$ & $\mathrm{Ga}$ \\
\hline \multicolumn{11}{|c|}{ Type } \\
\hline Z100-4 & High-Al & 5.81 & 1913 & 1300 & 1797 & 296 & 1769 & 0.00 & 828 & 64.80 \\
\hline Z100-5 & High-Al & 5.47 & 1871 & 1309 & 1754 & 301 & 1999 & 2.82 & 800 & 68.70 \\
\hline Z100-6 & High-Al & 6.86 & 1846 & 1282 & 1783 & 292 & 1939 & 2.19 & 767 & 64.40 \\
\hline Z100-7 & High-Al & 6.26 & 1764 & 1279 & 1804 & 293 & 1970 & 2.66 & 791 & 66.40 \\
\hline Z100-8 & High-Al & 5.14 & 1888 & 1318 & 1724 & 294 & 2058 & 3.39 & 794 & 68.00 \\
\hline Z100-9 & High-Al & 5.58 & 1923 & 1358 & 1755 & 301 & 2130 & 3.12 & 821 & 71.50 \\
\hline Z100-10 & High-Al & 4.84 & 1929 & 1332 & 1825 & 299 & 1888 & 0.00 & 781 & 64.80 \\
\hline Z100-11 & High-Al & 5.58 & 1961 & 1371 & 1747 & 303 & 2055 & 1.53 & 866 & 72.40 \\
\hline Z100-12 & High-Al & 4.89 & 1873 & 1342 & 1839 & 303 & 1915 & 1.04 & 879 & 69.70 \\
\hline Z105a-1 & High-Al & 5.89 & 1913 & 1351 & 1755 & 296 & 2138 & 3.12 & 823 & 68.10 \\
\hline Z105a-2 & High-Al & 5.98 & 1852 & 1367 & 1743 & 302 & 2207 & 0.00 & 836 & 72.50 \\
\hline Z105a-3 & High-Al & 5.02 & 1942 & 1344 & 1714 & 308 & 2127 & 1.85 & 858 & 67.10 \\
\hline Z105a-4 & High-Al & 6.10 & 1846 & 1333 & 1789 & 301 & 1844 & 1.68 & 869 & 70.20 \\
\hline Z105a-5 & High-Al & 5.08 & 1908 & 1346 & 1816 & 297 & 1949 & 2.30 & 824 & 67.90 \\
\hline Z105a-6 & High-Al & 3.57 & 1972 & 1385 & 1742 & 309 & 2157 & 1.33 & 895 & 66.80 \\
\hline Z105a-7 & High-Al & 6.14 & 1884 & 1352 & 1778 & 303 & 2137 & 4.95 & 811 & 68.30 \\
\hline Z105a-8 & High-Al & 6.13 & 1954 & 1369 & 1763 & 308 & 2173 & 2.81 & 856 & 72.00 \\
\hline Z105a-9 & High-Al & 6.18 & 1880 & 1348 & 1779 & 304 & 2108 & 3.04 & 844 & 69.80 \\
\hline Z105a-10 & High-Al & 5.59 & 1944 & 1386 & 1771 & 308 & 2135 & 2.82 & 863 & 70.70 \\
\hline Z105a-11 & High-Al & 6.15 & 1857 & 1366 & 1780 & 308 & 2035 & 2.63 & 861 & 73.10 \\
\hline Z105a-12 & High-Al & 5.50 & 1911 & 1388 & 1798 & 307 & 2179 & 1.99 & 850 & 70.90 \\
\hline Z105a-13 & High-Al & 5.03 & 1943 & 1352 & 1790 & 300 & 1981 & 0.00 & 789 & 67.20 \\
\hline ST DEV & & 0.71 & 47.43 & 32 & 39 & 6 & 134.74 & 0.91 & 36.66 & 2.88 \\
\hline Average & & 5.49 & 1896 & 1342 & 1778 & 301 & 2018.1 & 2.45 & 830.3 & 68.88 \\
\hline $\mathrm{Z} 103 \mathrm{c}-1$ & High-Al & 6.76 & 1836 & 984 & 1777 & 302 & 1927 & 6.01 & 678 & 60.80 \\
\hline $\mathrm{Z} 103 \mathrm{c}-2$ & High-Al & 6.17 & 1854 & 960 & 1745 & 300 & 1944 & 5.06 & 657 & 60.50 \\
\hline$Z 103 c-3$ & High-Al & 5.93 & 1889 & 964 & 1800 & 301 & 1965 & 6.16 & 587 & 57.30 \\
\hline$Z 103 c-4$ & High-Al & 5.85 & 1849 & 984 & 1757 & 306 & 1999 & 5.95 & 616 & 60.50 \\
\hline$Z 103 c-5$ & High-Al & 5.66 & 1865 & 967 & 1792 & 303 & 1971 & 6.32 & 644 & 61.50 \\
\hline$Z 103 c-6$ & High-Al & 6.02 & 1830 & 967 & 1721 & 307 & 1999 & 5.57 & 616 & 58.70 \\
\hline $\mathrm{Z} 103 \mathrm{c}-7$ & High-Al & 6.29 & 1843 & 965 & 1750 & 300 & 2008 & 3.70 & 615 & 60.90 \\
\hline$Z 103 c-8$ & High-Al & 6.19 & 1863 & 967 & 1809 & 310 & 1883 & 5.14 & 646 & 57.90 \\
\hline$Z 103 c-9$ & High-Al & 5.94 & 1856 & 981 & 1793 & 306 & 1949 & 4.27 & 599 & 60.40 \\
\hline$Z 103 c-10$ & High-Al & 5.45 & 1865 & 984 & 1790 & 314 & 1963 & 10.20 & 634 & 62.80 \\
\hline Z103c- 11 & High-Al & 5.47 & 1818 & 952 & 1758 & 317 & 1913 & 6.04 & 613 & 58.20 \\
\hline Z103c - 12 & High-Al & 5.79 & 1856 & 972 & 1746 & 301 & 2076 & 6.88 & 646 & 60.40 \\
\hline$Z 103 c-13$ & High-Al & 5.63 & 1858 & 982 & 1880 & 333 & 1820 & 4.56 & 642 & 58.30 \\
\hline$Z 103 c-14$ & High-Al & 5.56 & 1870 & 984 & 1774 & 306 & 2030 & 6.93 & 627 & 59.00 \\
\hline$Z 103 c-15$ & High-Al & 6.37 & 1858 & 977 & 1768 & 307 & 2044 & 7.01 & 642 & 59.80 \\
\hline$Z 103 c-16$ & High-Al & 4.95 & 1874 & 982 & 1745 & 314 & 1935 & 1.51 & 641 & 61.60 \\
\hline$Z 103 c-18$ & High-Al & 5.74 & 1894 & 983 & 1796 & 308 & 1985 & 6.38 & 641 & 60.60 \\
\hline Z103c- 19 & High-Al & 4.95 & 1898 & 987 & 1922 & 339 & 1704 & 2.52 & 632 & 57.70 \\
\hline$Z 103 c-20$ & High-Al & 6.38 & 1871 & 970 & 1783 & 312 & 1869 & 3.87 & 646 & 59.40 \\
\hline Z103c - 21 & High-Al & 6.65 & 1933 & 988 & 1827 & 317 & 1833 & 5.40 & 607 & 60.00 \\
\hline ST DEV & & 0.48 & 25.81 & 10 & 47 & 10 & 87.05 & 1.84 & 21.47 & 1.46 \\
\hline Average & & 5.88 & 1864 & 975 & 1787 & 310 & 1940.9 & 5.47 & 631.5 & 59.81 \\
\hline
\end{tabular}




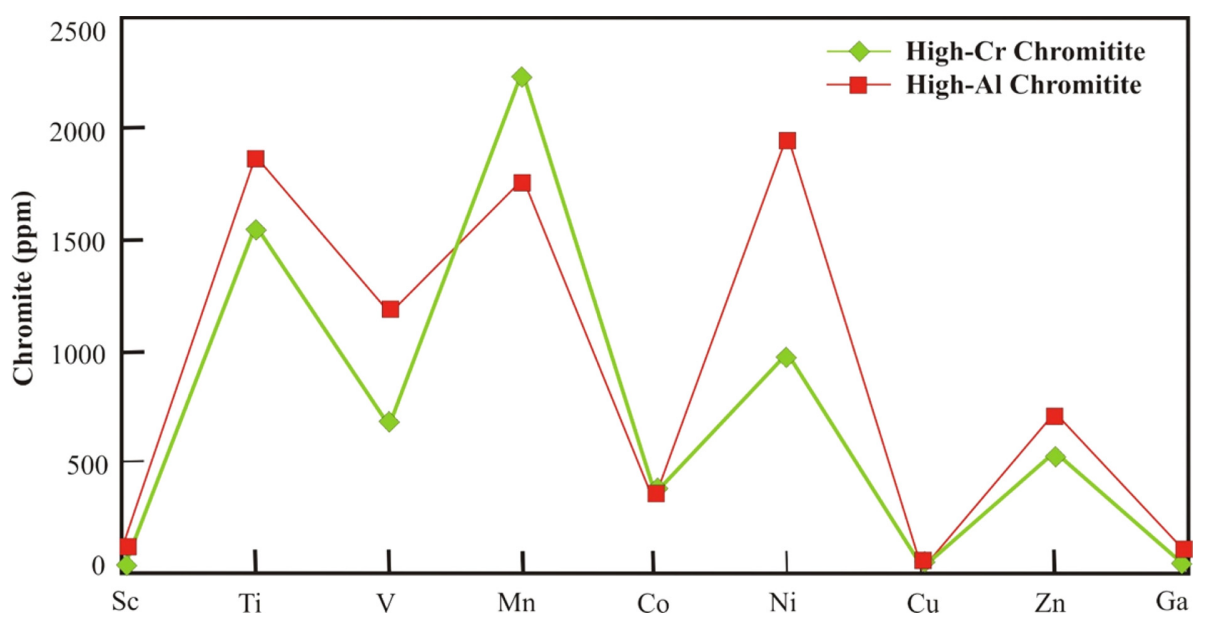

Figure 7. Comparison of average trace elements concentrations in high-Cr and high-Al chromitites, showing distinct differences between the two types.

ments contents in amphibole range between 0.92 to $2.80 \mathrm{wt} \%$ for $\mathrm{Na}_{2} \mathrm{O}$, 0.01 to $0.03 \mathrm{wt} \%$ for $\mathrm{K}_{2} \mathrm{O}$ and 0.17 to $0.79 \mathrm{wt} \%$ for $\mathrm{TiO}_{2}$.

\section{Discussion \\ Nature of Parental Melts of the Zhob Valley Chromitite}

The $\mathrm{Al}_{2} \mathrm{O}_{3}$ and $\mathrm{TiO}_{2}$ (wt $\%$ ) contents of chromium spinel are characteristics for determining the origin and tectonic setting of parental melt of chromitites (Auge, 1987; Melcher et al., 1997; Kamenetsky et al., 2001; Uysal et al., 2007a; Rollinson, 2008; Page and Barnes, 2009; Zaccarini et al., 2011). The parental melts occurring chromitites are formed by various processes such as partial melting (Kelemen et al., 1992; Arai, 1994; Zhou et al., 1996), melt/rock reaction and melt/melt interaction (Zhou et al., 1998; Melcher et al., 1999; Kamenetsky et al., 2001; Uysal et al., 2005, 2009). Several authors noted that the concentration of $\mathrm{Al}_{2} \mathrm{O}_{3}$ $(\mathrm{wt} \%)$ and $\mathrm{TiO}_{2}(\mathrm{wt} \%$ ), and $\mathrm{FeO} / \mathrm{MgO}$ ratios of chromium spinel are

Table 3. Composition of olivine and amphibole inclusions in chromite. Ol=Olivine and Amp=Amphibole, $N=$ Number of spot analysis, bdl= below detection limit

\begin{tabular}{|c|c|c|c|c|c|c|c|c|c|c|c|}
\hline Sample No & B43 & $\bar{B} 43 \mathrm{a}$ & B44 cr & B63 cr & KO30 & $\overline{Z Z 100}$ & $\overline{Z Z 100 c}$ & $\overline{Z Z 103 c}$ & B43 & KO30 & $\overline{Z Z 100}$ \\
\hline Mineral & $\mathrm{Ol}$ & $\mathrm{Ol}$ & $\mathrm{Ol}$ & $\mathrm{Ol}$ & $\mathrm{Ol}$ & $\mathrm{Ol}$ & $\mathrm{Ol}$ & $\mathrm{Ol}$ & Amp & Amp & Amp \\
\hline Type & High-Cr & High-Cr & High-Cr & High-Cr & High-Cr & High-Al & High-Al & High-Al & High-Cr & High-Cr & High-Al \\
\hline $\mathrm{N}$ & 4 & 1 & 3 & 1 & 2 & 3 & 2 & 1 & 2 & 1 & 2 \\
\hline $\mathrm{SiO}_{2}$ & 42.55 & 41.50 & 41.59 & 41.99 & 42.93 & 43.06 & 42.97 & 42.51 & 52.41 & 51.08 & 46.89 \\
\hline $\mathrm{TiO}_{2}$ & bdl & bdl & 0.01 & 0.04 & 0.02 & 0.26 & bdl & 0.03 & 0.17 & 0.79 & 0.70 \\
\hline $\mathrm{Al}_{2} \mathrm{O}_{3}$ & 0.22 & 0.78 & 0.54 & bdl & 0.75 & 0.06 & 0.57 & 0.19 & 6.55 & 7.04 & 11.76 \\
\hline $\mathrm{Cr}_{2} \mathrm{O}_{3}$ & 0.29 & 0.27 & 0.35 & 0.62 & 0.36 & 0.64 & 0.41 & 0.80 & 1.76 & 0.90 & 1.70 \\
\hline $\mathrm{FeO}$ & 3.25 & 3.62 & 2.70 & 2.88 & 3.21 & 3.48 & 3.38 & 2.86 & 3.35 & 1.60 & 2.08 \\
\hline $\mathrm{MnO}$ & 0.50 & bdl & 0.20 & bdl & 0.27 & 0.10 & 0.18 & 0.30 & bdl & 0.04 & 0.02 \\
\hline $\mathrm{NiO}$ & 0.80 & bdl & 0.28 & 0.69 & 0.05 & 0.21 & 0.05 & 0.50 & 0.15 & 0.30 & 0.15 \\
\hline $\mathrm{MgO}$ & 52.76 & 52.92 & 53.51 & 54.53 & 51.88 & 51.85 & 51.76 & 52.25 & 21.19 & 21.02 & 20.01 \\
\hline $\mathrm{CaO}$ & bdl & bdl & 0.03 & 0.02 & 0.06 & 0.15 & 0.05 & 0.07 & 11.48 & 12.07 & 12.11 \\
\hline $\mathrm{Na}_{2} \mathrm{O}$ & 0.36 & bdl & bdl & bdl & bdl & 0.06 & bdl & bdl & 0.92 & 1.40 & 2.80 \\
\hline $\mathrm{K}_{2} \mathrm{O}$ & 0.05 & bdl & 0.04 & bdl & 0.03 & 0.28 & 0.04 & 0.40 & 0.01 & 0.02 & 0.03 \\
\hline Total $\sum$ & 99.46 & 99.10 & 99.24 & 100.78 & 99.12 & 99.90 & 99.33 & 99.92 & 97.88 & 96.24 & 98.25 \\
\hline \multicolumn{12}{|c|}{ Cations (structural formula on the basis of 4 oxygen for olivine and 23 oxygen for amphibole) } \\
\hline $\mathrm{Si}$ & 1.02 & 1.00 & 1.00 & 0.99 & 1.03 & 1.03 & 1.03 & 1.01 & 7.25 & 7.14 & 6.53 \\
\hline $\mathrm{Ti}$ & 0.00 & 0.00 & 0.00 & 0.00 & 0.00 & 0.00 & 0.00 & 0.00 & 0.01 & 0.08 & 0.07 \\
\hline $\mathrm{Al}$ & 0.00 & 0.02 & 0.02 & 0.00 & 0.01 & 0.01 & 0.02 & 0.01 & 1.07 & 1.16 & 1.93 \\
\hline $\mathrm{Cr}$ & 0.00 & 0.01 & 0.01 & 0.01 & 0.01 & 0.01 & 0.01 & 0.02 & 0.19 & 0.10 & 0.19 \\
\hline $\mathrm{Fe}^{2+}$ & 0.07 & 0.07 & 0.05 & 0.06 & 0.06 & 0.07 & 0.07 & 0.06 & 0.39 & 0.19 & 0.24 \\
\hline $\mathrm{Mn}$ & 0.00 & 0.00 & 0.00 & 0.00 & 0.01 & 0.00 & 0.00 & 0.01 & 0.00 & 0.00 & 0.00 \\
\hline $\mathrm{Ni}$ & 0.01 & 0.00 & 0.00 & 0.01 & 0.00 & 0.00 & 0.00 & 0.01 & 0.01 & 0.01 & 0.01 \\
\hline $\mathrm{Mg}$ & 1.88 & 1.89 & 1.91 & 1.92 & 1.85 & 1.84 & 1.84 & 1.86 & 4.37 & 4.38 & 4.16 \\
\hline $\mathrm{Ca}$ & 0.00 & 0.00 & 0.00 & 0.00 & 0.00 & 0.00 & 0.00 & 0.00 & 1.70 & 1.81 & 1.81 \\
\hline $\mathrm{Na}$ & 0.01 & 0.00 & 0.00 & 0.00 & 0.00 & 0.00 & 0.00 & 0.00 & 0.25 & 0.38 & 0.76 \\
\hline $\mathrm{K}$ & 0.00 & 0.00 & 0.00 & 0.00 & 0.00 & 0.00 & 0.00 & 0.01 & 0.00 & 0.00 & 0.01 \\
\hline Total $\Sigma$ & 2.98 & 2.99 & 2.99 & 2.99 & 2.96 & 2.96 & 2.96 & 2.97 & 15.22 & 15.25 & 15.70 \\
\hline $\mathrm{Mg \#}$ & 0.96 & 0.96 & 0.97 & 0.97 & 0.97 & 0.96 & 0.96 & 0.97 & 0.92 & 0.96 & 0.95 \\
\hline
\end{tabular}


in direct relation with the composition of the parental melts (Kamenetsky et al., 2001; Rollinson, 2008).

The equation developed by Rollinson (2008) based on the data of chromite spinels from 36 different igneous suites from oceanic, arc, and intraplate tectonic setting of Kamenetsky et al. (2001), can be used to derive the composition of parental melts:

$$
\begin{aligned}
& \mathrm{Al}_{2} \mathrm{O}_{3 \_ \text {melt }}=5.2181 * \mathrm{Ln}\left(\mathrm{Al}_{2} \mathrm{O}_{3 \_ \text {chromite }}\right)-1.50505 \\
& \mathrm{Ln}\left(\mathrm{TiO}_{2 \_ \text {melt }}\right)=1.0963 *\left(\mathrm{TiO}_{2 \_ \text {chromite }}\right){ }^{0.7863}
\end{aligned}
$$

The calculated $\mathrm{Al}_{2} \mathrm{O}_{3}\left(\mathrm{wt} \%\right.$ ) and $\mathrm{TiO}_{2}(\mathrm{wt} \%)$ compositions of the parental melts of both high-Cr and high-Al chromitites from the Zhob valley ophiolite are presented in Table 1 . The concentrations of $\mathrm{Al}_{2} \mathrm{O}_{3}$ of the parental melts of high-Cr chromitites ranges from 9.2 to 13.8 $\mathrm{wt} \%$, while for the high- $\mathrm{Al}$ ones it varies between 14.9 and $15.4 \mathrm{wt} \%$. Furthermore, the $\mathrm{TiO}_{2}$ concentration of parental melts of high-Cr and high-Al chromitites varies from 0.02 to $0.53 \mathrm{wt} \%$ and from 0.17 to $0.42 \mathrm{wt} \%$, respectively. The $\mathrm{Al}_{2} \mathrm{O}_{3}$ contents of the inferred parental melts of high-Cr chromitites (9.2-13.8 wt\%) are in agreement with $\mathrm{Al}_{2} \mathrm{O}_{3}$ concentrations of chromitites of the Elekdag Ophiolite (northern Turkey) (9.4-13.2 wt\%; Dönmez et al., 2014), Kop Mountain (Turkey) (10.1-13.5 wt\%; Uysal et al., 2007a), Thetford Mines Ophiolite (9.2-12.9 wt\%; Page and Barnes, 2009), Oman Ophiolite (11.812.9 wt\%; Rollinson, 2008), and Luobusa Ophiolite (13.8 wt\%; Zhou et al., 2014). In addition, the $\mathrm{Al}_{2} \mathrm{O}_{3}$ (wt\%) values of high-Cr chromitites fall within the range of composition of chromite derived from boninite melt (10.6-14.4 wt\%; Wilson, 1989). However, the calculated

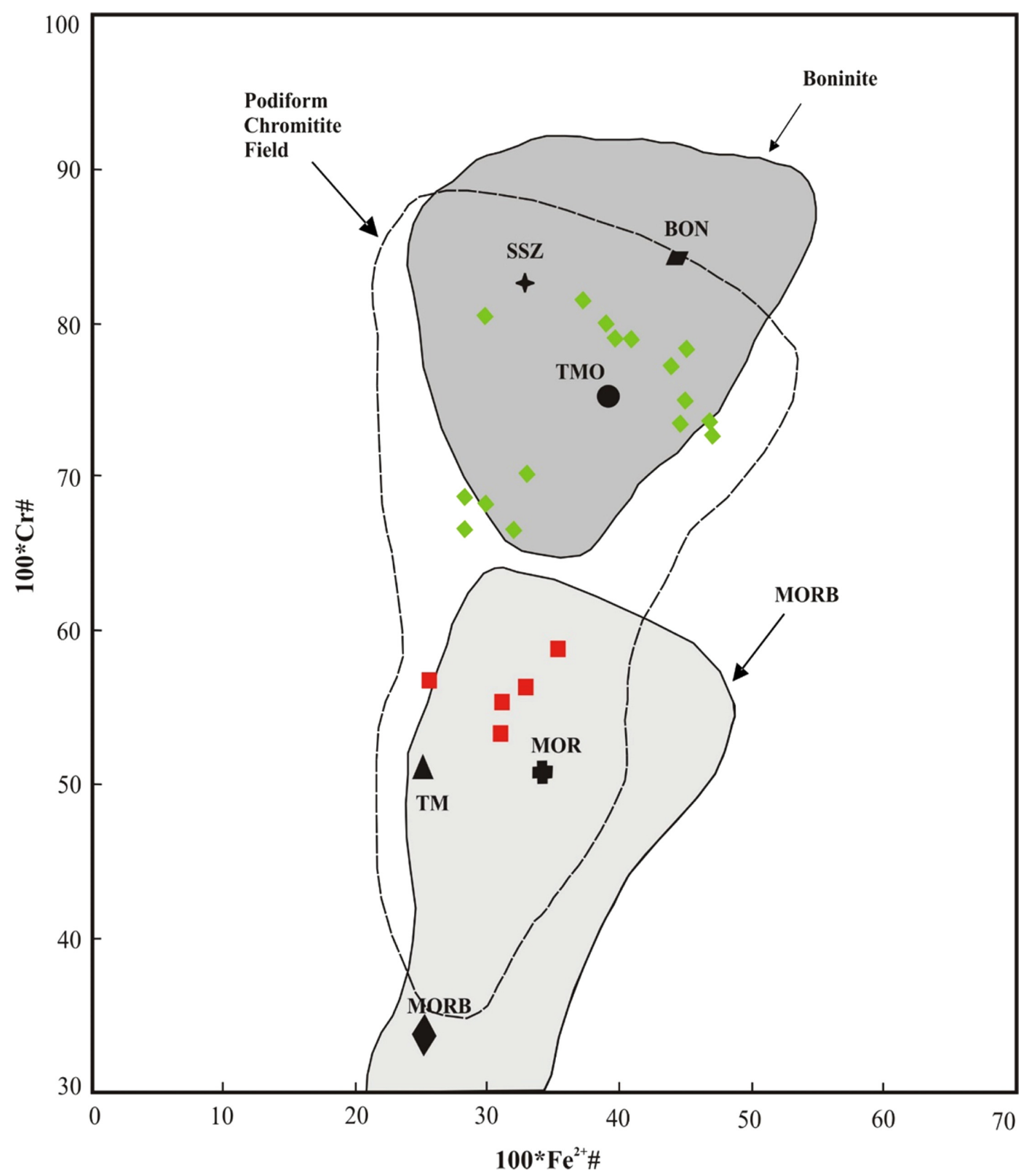

Figure $8.100 * \mathrm{Cr} \#$ vs. $\mathrm{Fe}^{2+}$ \# for chromite from podiform chromitites in the Zhob valley ophiolite. Boninite and MORB values taken from Page and Barnes, (2009). Fields for chromite from MORB and boninite are from Barnes and Roeder (2001). Hess Deep (a MOR setting) (Arai and Matsukage, 1998) and Japan Arc Xenolith (a supra-subduction setting) (Arai and Abe, 1994), Tari-Misaka (Arai and Yurimoto, 1994), TMO (Page and Barnes, 2009). Average contents of TMO, Tr and TM are plotted. MOR=Mid ocean ridge, SSZ=Supra-subduction zone, Tr= Turkish deposits, TM, Tari-Misaka, TMO=Thetford Mines Ophiolite. 
$\mathrm{Al}_{2} \mathrm{O}_{3}$ (wt\%) values of the parental magma of studied high-Al chromitites (14.9-15.4 wt\%) are similar to the high-Al chromitites of southern Turkey (14.5-16.2 wt\%; Akmaz et al., 2014), Sagua de Tanamo (eastern Cuba) (15-16 wt\%; Gonzalez-Jimenez et al., 2011), KempirsaiBatamshinsk (Kazakhstan) (13.5-16.7 wt\%; Melcher et al., 1997), and Sartohay Ophiolite (16.3 wt \%; Zhou et al., 2014) and comparable to the $\mathrm{Al}_{2} \mathrm{O}_{3}$ (wt\%) concentrations with MORB melts ( $16 \mathrm{wt} \%$; Wilson,

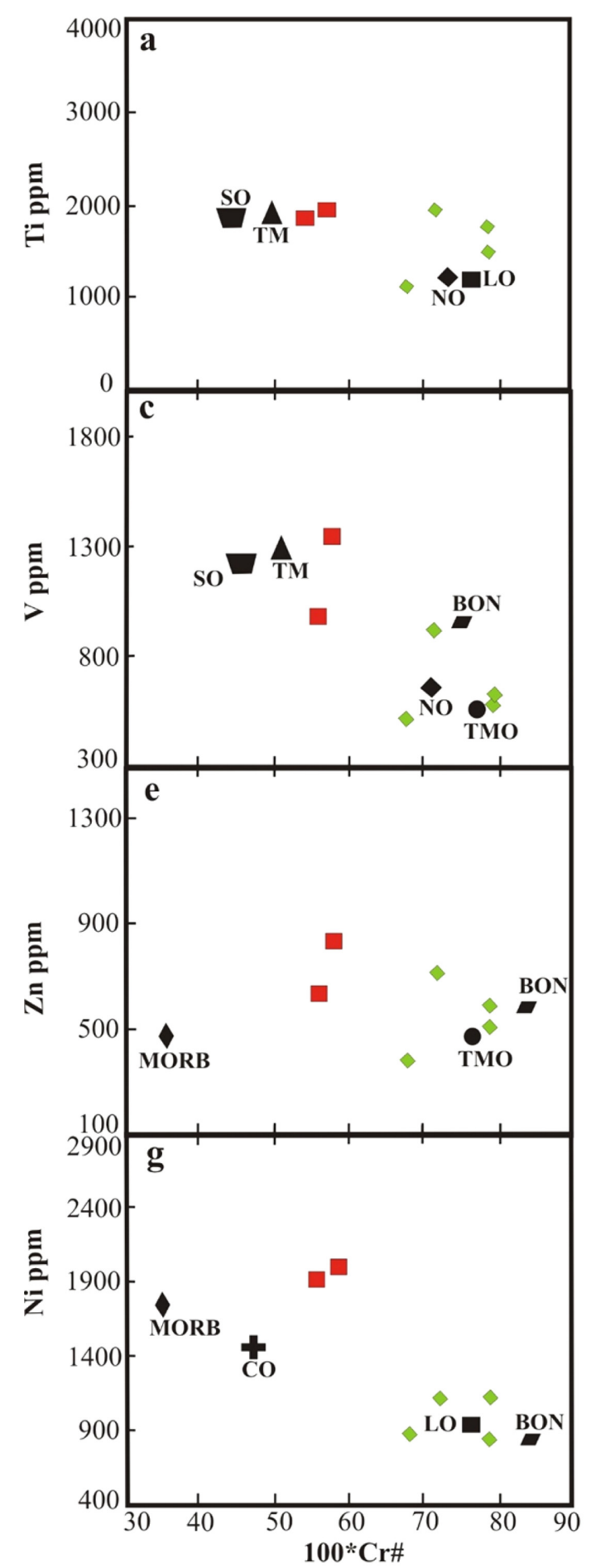

1989). Thus, the data indicates that parental magma of high-Cr chromitites were boninitic while high-Al chromitite was crystallized from MORB-like melts.

In the $\mathrm{Fe}^{2+} \#$ vs $\mathrm{Cr} \#$ diagram, chromitites from the Zhob valley ophiolite plot in the high-Cr and high-Al fields of podiform chromitites, which further extends from the high-Cr boninitic field to the high-Al MORB field (Fig. 8). With the exception of a few samples that have

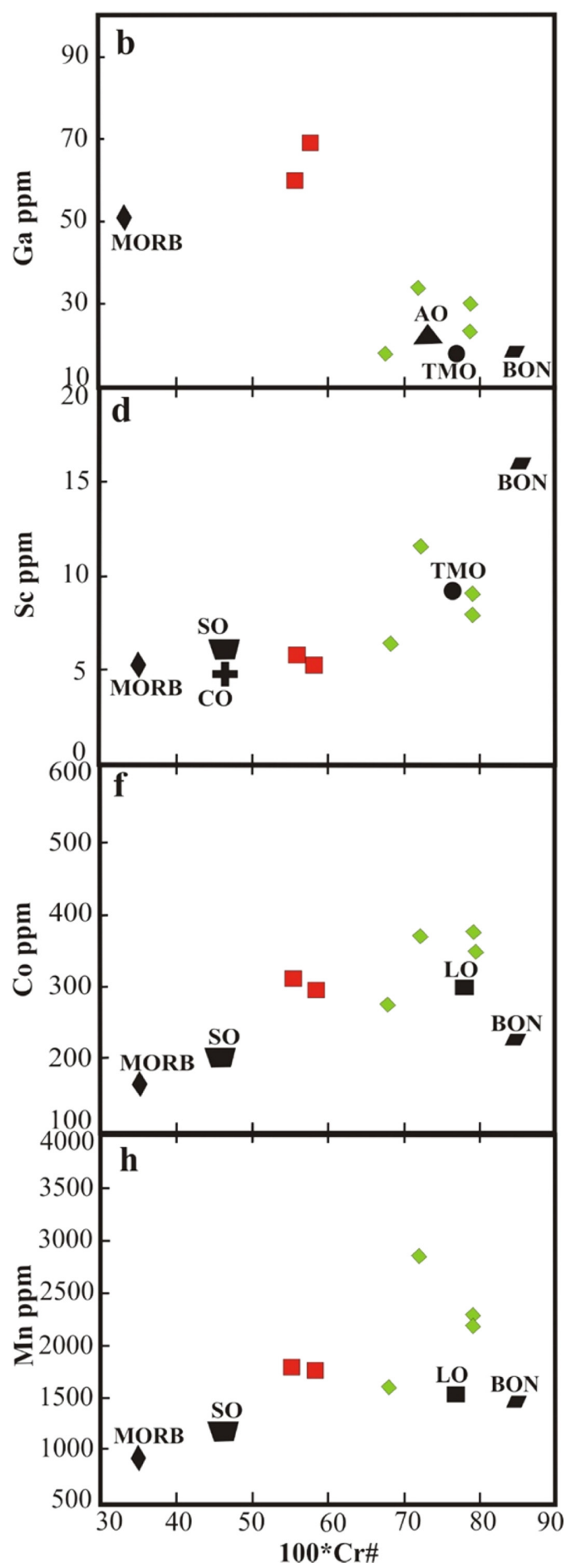

Figure 9. (a) Ti vs. Cr\#, (b) Ga vs. C\#, (c) V vs. Cr\#, (d) Sc vs. Cr\#, (e) Zn vs. Cr\#, (f) Co vs. Cr\#, (g) Ni vs. Cr\#, (h) Mn vs. Cr\# for chromites from Zhob valley ophiolite. LO=Luobusa Ophiolite (Zhou et al., 2014), SO=Sartohay Ophiolite (Zhou et al., 2014), AO=Acoje Ophiolite (Zhou et al., 2014), CO=Coto Ophiolite (Zhou et al., 2014). Podiform chromitite, MORB and BON fields, other data source and acronyms are as in Figure 8. Note that average contents of elements are plotted. 
higher $\mathrm{Fe}^{2+} \#$, high-Cr chromitites of the Zhob valley ophiolite exhibit an identical range of compositions to the Thetford Mines Ophiolite (TMO), supra-subduction zone (SSZ), and high-Cr chromite from Troodos Ophiolite (Fig. 8) (Arai and Abe, 1994; Arai and Yurimoto, 1994; Panktunc and Cabri, 1995; Page and Barnes, 2009). The compositions of high-Al chromites shows a similar range to the high-Al chromitites from the Tari-Misaka Complex (TM), and MOR (Auge, 1987; Arai and Yurimoto, 1994; Ahmed and Arai, 2002).

$\mathrm{Ga}, \mathrm{Ti}, \mathrm{Zn}, \mathrm{Ni}$ and $\mathrm{V}$ concentrations of chromite from the Zhob valley chromitites show negative correlation with $\mathrm{Cr} \#$ (Fig. 9) which reflects crystal fractionation in the parental melt of chromite (Page and Barnes, 2009). High-Cr chromitites from the Zhob valley ophiolite have a similar range of Ti contents to the northern Oman (NO), Luobusa Ophiolite (LO), and chromitites from supra-subduction zone, while high-Al chromitites have similar contents of Ti to the Tari-Misaka Complex (TM) and Sartohay Ophiolite (SO) (Fig. 9a). Gallium, vanadium and nickel show a more pronounced inverse correlation with $\mathrm{Cr} \#$ than $\mathrm{Ti}$ and $\mathrm{Zn}$ (Fig. 9). Ga in high-Cr chromitites is similar with the TMO and Acoje ophiolite (AO) and close to chromites in boninites (Fig. 9b), while high-Al chromitites are enriched in Ga contents compared to those of the Sartohay Ophiolite and close to MORB content. Vanadium in high-Cr chromitites exhibits a similar range to the TMO and northern Oman chromitites while it is lower than chromites in boninites. High-Al chromitites are in agreement with TM and Sartohay chromitites in terms of vanadium contents (Fig. 9c). The depletion of $\mathrm{V}$ in high$\mathrm{Cr}$ chromitite is believed to be the result of various oxidation conditions of boninitic parental melts (Page and Barnes, 2009). It is partly related to oxidizing conditions that vary from the mantle to the top of the crust or to oxidation of boninite magmas during shallow ascent and eruption (Page and Barnes, 2009).

Sc contents of high-Cr and high-Al chromitites show similar ranges to the TMO and boninite and Sartohay, Coto Ophiolites (CO) and MORB, respectively (Fig. 9d). In high $\mathrm{Cr}$ chromitites, $\mathrm{Zn}$ and Ni contents are comparable with boninite, TMO and LO, but Co and Mn are higher than boninite (Fig. 9e, f, g, h). On the other hand, high-Al chromitite is enriched in $\mathrm{Ni}, \mathrm{Mn}, \mathrm{Zn}$ and $\mathrm{Co}$ as compared to those from MORB (Fig. 9e, f, g, h). Especially, Mn, Zn and Co enrichments are interpreted as metamorphism of chromitites in the literature (Colâs et al., 2014; Uysal et al., 2018). Similarly high concentrations of these elements are reported from highly altered chromite from Morocco (Gahlan and Arai, 2007), Greece (Paraskevopoulos and Economou, 1981; Michailidis, 1990; Economou-Eliopoulos, 2003), Dobromirtsi Chromitites, Bulgaria (Gonzalez-Jimenez el al., 2015) and some deposits from southeastern Turkey (Akmaz et al., 2014).

Trace elements of chromitites from the Zhob valley ophiolite were normalized to chromite compositions from MORB and arranged on the pattern of Page and Barnes (2009) in Figure 10. Moreover, high$\mathrm{Cr}$ chromitites were compared to Luobusa Ophiolite (LO) (Zhou et al., 2014) and Thetford Mines Ophiolite (TMO) (Page and Barnes, 2009) whereas high-Al chromitites were compared to Sartohay Ophiolite (SO) (Zhou et al., 2014) and Zambales Ophiolite (ZO) (Gonzalez-Jimenez et al., 2012) (Fig. 10). With minor exceptions, concentrations of almost all elements of high-Cr chromitite are in agreement with TMO while they are partially enriched in $\mathrm{Ga}, \mathrm{Ti}, \mathrm{Zn}$, and Mn compared with Luobusa Ophiolite (Fig. 10a). The high-Al chromitites show a similar pattern to the Zambales Ophiolite (ZO) and Sartohay Ophiolite (SO).
However, they are slightly enriched in $\mathrm{Zn}, \mathrm{Co}, \mathrm{Mn}(\mathrm{ppm})$ and $\mathrm{Cr}_{2} \mathrm{O}_{3}$ (wt\%) and depleted in $\mathrm{Al}_{2} \mathrm{O}_{3}$ (wt\%), Ti (ppm) and $\mathrm{MgO}$ (wt\%) compared with those in $\mathrm{ZO}$ and higher in terms of $\mathrm{Ga}, \mathrm{Ni}, \mathrm{Zn}, \mathrm{Co}, \mathrm{Mn}$ (ppm) and $\mathrm{Cr}_{2} \mathrm{O}_{3}$ (wt\%) compared to these elements in SO (Fig. 10b). The $\mathrm{Ti}, \mathrm{V}, \mathrm{Ni}, \mathrm{Zn}$ and $\mathrm{Ga}$ contents in high-Al chromitites more enriched than high-Cr ones suggest that parental melts from which high-Al chromitites were formed. The similarity of high-Cr chromitite from studied deposits to the boninite and TMO indicates genetic link between them and suggests that high-Cr chromitites from Zhob valley ophiolite crystallized out from boninitic melts. On the other hand, high-Al chromitites exhibit similar patterns to the MORB. But high-Al chromitite is slightly depleted in $\mathrm{Al}_{2} \mathrm{O}_{3}$ and Ti compared to those from MORB while similar to those from Sartohay Ophiolite which reveals high-Al chromitites have thoiilitic/MORB affinity.

\section{Compositional Variation and Alteration in Chromites}

The profile analyses of chromite grains of both types of chromitites show that most of them are unzoned. However, in some grains of high-Cr chromitite, $\mathrm{Cr}$ contents decrease while $\mathrm{Al}_{2} \mathrm{O}_{3}$ (wt\%) and $\mathrm{FeO}_{\text {total }}$ (wt $\%$ ) contents increase from core to rim. Therefore, the $\mathrm{Cr} \#$ values are decreasing from core to rim in high-Cr chromite grains (Fig. 6b). This kind of zoning is considered to be the results of fractionation of melt during the formation of chromite, hence, reflect magmatic zoning (Akmaz et al., 2014). On the other hand, in some grains of high-Al chromitites, $\mathrm{Al}_{2} \mathrm{O}_{3}(\mathrm{wt} \%)$ and $\mathrm{MgO}$ (wt $\%$ ) contents decrease from core to rim while $\mathrm{Cr}$ and $\mathrm{FeO}_{\text {total }}(\mathrm{wt} \%)$ contents increase from core to rim, resulting in an increase of $\mathrm{Cr} \#$ from core to rim (Fig. 6a). This kind of zonation indicates that secondary processes had affected the chromite grains which had resulted in the formation of ferritchromite.

The alteration process observed in ophiolitic chromitites is associated with many events which are serpentinization (Burkhard, 1993), regional metamorphism (Barnes, 2000; González-Jiménez et al., 2009), decomposition (Pooley, 2004) and lateritization (Economou-Eliopoulos, 2003). As a result of petrographic investigations, any decomposition and lateritization process was not observed in Zhob valley chromitites. Therefore, the main cause of ferritchromite formation can be explained as serpentinization and/or metamorphism (Kapsiotis, 2015).

The formation of ferritchromite in the Kalkan chromitites (southern Urals), has been described and three stages were suggested for its development (Merlini et al., 2009). In first stage chromite grains equilibrated with surrounding olivine. The second stage involved the serpentinization (lizardite and chrysotile) of the matrix rich with olivine at approximately 200 to $300{ }^{\circ} \mathrm{C}$ (Auzende et al., 2006; Hajialioghli et al., 2007). Almost all lizardite/chrysotile transformed by prograde metamorphism at more than $300{ }^{\circ} \mathrm{C}$ into the polymorph antigorite in the third stage. The metamorphic reaction $\left.2\left(\mathrm{Mg}_{0.60} \mathrm{Fe}_{0.40}\right)\left(\mathrm{Cr}_{1.30} \mathrm{Al}_{0.70}\right) \mathrm{O}_{4}\right)$ [chromite] + 3/2 $\left(\mathrm{Mg}_{2.57} \mathrm{Al}_{0.32} \mathrm{Fe}_{0.11}\right) \mathrm{Si}_{2} \mathrm{O}_{5}(\mathrm{OH})_{4}+\mathrm{H}_{2} \mathrm{O}+1 / 12 \mathrm{O}_{2}$ [antigorite $\rightarrow 7 / 6\left(\mathrm{Mg}_{0.40} \mathrm{Fe}_{0.60}\right)\left(\mathrm{Cr}_{1.85} \mathrm{Fe}_{0.08} \mathrm{Al}_{0.07}\right) \mathrm{O}_{4}$ [Fe-chromite] $+1 / 2$

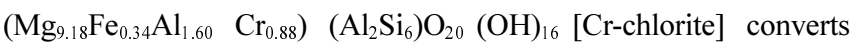
chromite-serpentine into ferritchromite-chromian-chlorite assemblages in the presence of a hydrous fluid phase (Merlini et al., 2009). Thus, according to this reaction, the generation of ferritchromite in chromite grains of high-Al chromitite from the Zhob valley ophiolite indicates that this is due to metamorphic processes (Barnes, 2000; Proenza et al., 2004; Mukherjee et al., 2010; Gervilla et al., 2012). 

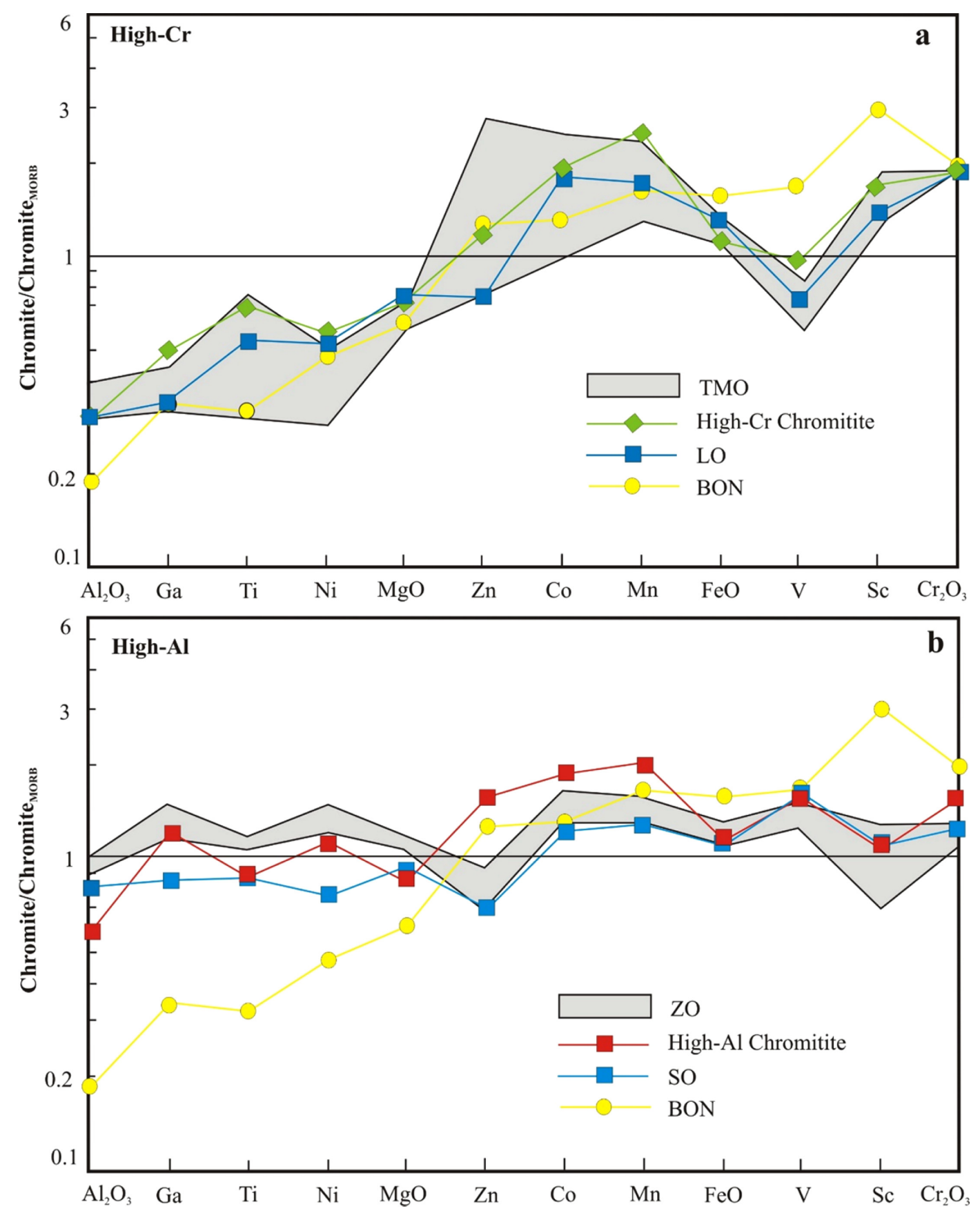

Figure 10. Chromite ${ }_{\text {MоRB }}$ normalized major-trace elements of high-Cr (a) and high-Al (b) Zhob valley ophiolitic chromitites. Data source for TMO and boninite values were from (Page and Barnes, 2009), ZO chromitites values from (Gonzalez-Jimenez et al., 2012) Luobusa and Sartohay chromitites values were from (Zhou et al., 2014). Data source and acronyms are as in Figure 8. Note that average contents of elements are plotted.

\section{Genesis and Geotectonic Environment}

Today, the formation of podiform chromitites is not fully understood. Fractional crystallization from melt supplied through magmatic conduits is regarded as the primary model for the formation of podiform chromitites (Thayer, 1964; Lebanc and Ceuleneer, 1992). Melt/rock reaction, multi stage partial melting, magma mixing and magma segregation processes can lead to chromite formation (Paktunc et al., 1990; Lebanc and Ceuleneer, 1992). These models consider that melt/rock reactions and eventually melt/melt mixing are significant processes in the genesis of podiform chromitites (Kelemen, 1990; Arai and Yurimoto, 1994; Zhou et al., 1996). A model proposed by Gonzales-Jimenez et al. (2014b) suggests that podiform chromitites form in networks and channels of highly porous dunite, which formed in the mantle peridotite by melt/rock reaction. In high permeability conduits, the mixing and crystallization of new basaltic magma yields a suitable environment for podiform chromitite formation. Size, type and texture of chromitites may indicate the pattern of melt flow at different melt/rock ratios within highly porous networks of channels and a range of temperature contrasts between melt and the host peridotite. 
Many researchers have revealed that both high-Cr and high-Al chromitites can occur together within depleted peridotite in the same ophiolitic massif as well as in separate massifs (Zhou et al., 1994; Leblanc, 1995; Melcher et al., 1997; Proenza et al., 1999; Ahmed et al., 2001; Gervilla et al., 2005; Uysal et al., 2007a, 2009; Zaccarini et al., 2011; Kapsiotis, 2013). In addition, several authors explained the coexistence of high-Cr and high-Al chromitites in different ways. 1- It has been suggested that the melt responsible for the formation of chromite in a supra-subduction zone environment origins from several depleted mantle sources (MORB to boninitic type melt) (Uysal et al., 2009; Gonzales-Jimenez et al. 2011; Zaccarini et al., 2011). 2- Much depletion of mantle peridotite upwards in mantle sequence, with enhancement of $\mathrm{Cr} \#$ values in ophiolitic chromitites situated in residual mantle (Leblanc and Nicolas, 1992; Stowe, 1994; Uysal et al., 2007b). Chromitites from the Zhob valley ophiolitic bodies have lenses and pods of variable size that have sharp contacts with their dunite envelopes, and interlayered dunite and chromitites. These are characteristics that support a model in which metasomatic-magmatic processes have occurred in a network of channels through which melts percolated and reacted with peridotite wall rocks.

Contents of $\mathrm{Al}_{2} \mathrm{O}_{3}$ and $\mathrm{TiO}_{2}$ in chromite are related to the $\mathrm{Al}_{2} \mathrm{O}_{3}$ and $\mathrm{TiO}_{2}$ contents of parental melts of chromites which provide useful indicators of tectonic settings (Kamenetsky et al., 2001). Calculated $\mathrm{Al}_{2} \mathrm{O}_{3}$ (wt\%) contents of parental melts of high-Cr and high-Al from Zhob valley ore bodies suggest that they have an arc and MORB geochemical affinity, respectively. The very low $\mathrm{TiO}_{2}$ content of parental melt of high-Cr chromitites suggests that the chromitites were derived from boninitic melt formed in an island arc environment while parental fluid composition of high-Al chromitites indicates that it has been derived from MORB melts.

Inclusions of silicate minerals in chromite grains have been reported in several podiform chromitites (Johan and Lebel, 1978; Johan et al., 1983; Augé, 1987; Lorand and Ceuleneer, 1989; Graham et al., 1996; Melcher et al., 1997; Gervilla et al., 2005). Some authors like Johan and Lebel (1978), and Johan et al. (1983) argue that hydrous inclusions are evidence of unmixing of basaltic melt and an aqueous fluid phase and other volatile constituents. Furthermore, hydrous silicates indicate that during the chromite crystallization basaltic melt was contaminated with a fluid phase (Watkinson and Mainwaring, 1980; Lorand and Ceuleneer, 1989). We have observed mineral inclusions in unaltered chromites of the Zhob valley. Chromite grains of both kinds of chromitites contain a variety of silicate mineral inclusions. These inclusions occur together in a single chromite grain as well as in separate grains. Among the silicate inclusion, olivine and amphibole are detected (Fig. $3 \mathrm{f}$ and h). These silicate inclusions are typically anhedral to subhedral in shape and granular in texture. These assemblages and textures of minerals indicate that the mineral inclusions represent crystallization products of trapped liquids during crystallization of chromite (Zhou et al., 2014). The presence of amphibole inclusions and high $\mathrm{Cr} \#$ values prove that high-Cr chromitites were formed by boninitic type melts in supra-subduction zone environment. However, minor-trace element composition and amphibole inclusions indicate that high-Al chromitites are generated by MORB-

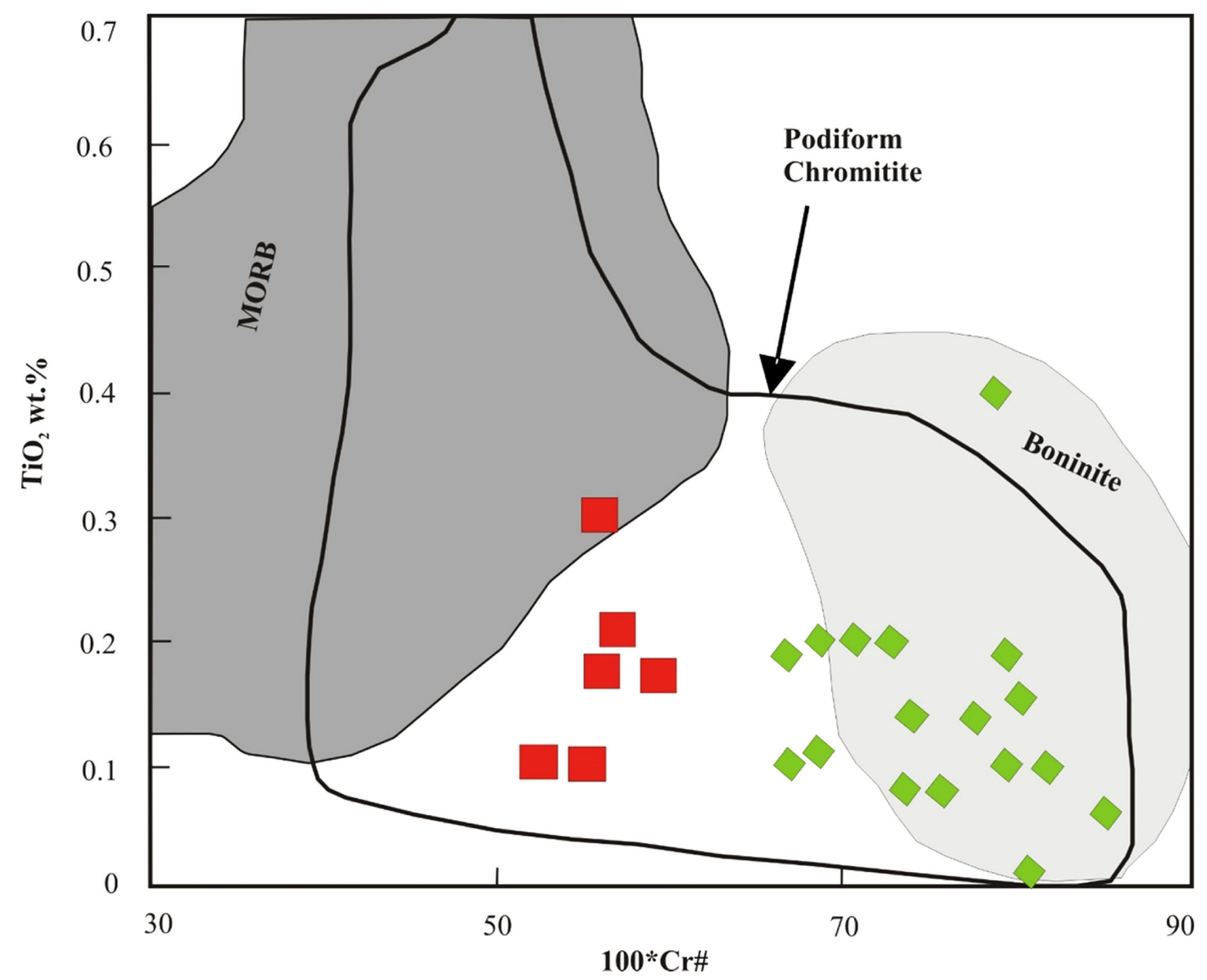

Figure 11. Tectonic discrimination using $100 * \mathrm{Cr} \#$ vs. $\mathrm{TiO}_{2}$ for chromites for podiform chromitites From Zhob valley ophiolite. The podiform chromitite field is from Page and Barnes (2009) and fields for chromite from MORB and boninite are from (Barnes and Roeder, 2001). 
like melts in back-arc setting.

$\mathrm{Cr} \#$ values and $\mathrm{TiO}_{2}$ contents (wt\%) of chromite from both chromitites are plotted in the diagram $\mathrm{TiO}_{2}$ vs $\mathrm{Cr} \#$ (Fig. 11). Both chromitites fall in the field of ophiolitic podiform chromitites while high-Cr chromitites show good similarities with chromitites that are derived from boninitic melts, generated in supra-subduction zones and high-Al chromitites analyses plot close to the MORB field.

Using the diagram $\left(\mathrm{Al}_{2} \mathrm{O}_{3}\right.$ vs $\left.\mathrm{TiO}_{2} \mathrm{wt} \%\right)$ of Kamenetsky et al. (2001) which is used to separate chromites crystallized from OIB, LIP, MORB and arc related basaltic melts (Fig. 12), high-Cr chromitites related the Zhob valley ophiolite exhibit a positive correlation and plot in or near to the arc field which suggests boninitic affinity. The high-Al chromitites plot within or close to the MORB field and display MORB affinity. Further, trace elements also impart suitable evidences for tectonic environments. Ga is compatible in chromian spinel (Malvin and Drake, 1987) and its concentration in chromites is a sensitive indicator of magma compositions and degree of depletion (Paktunc and Cabri, 1995, Zhou et al., 2014). Ga decreases with increasing Cr\# (Fig. 9b) (from high-Al to high-Cr chromitite) and inversely correlations with $\mathrm{Cr}$. Thus, this correlation reveals a switch from a mid-ocean ridge setting to a supra-subduction zone setting (Alabaster et al., 1982; Umino et al., 1990; Dare et al., 2008; Pagé and Barnes, 2009).

\section{Conclusions}

In the Zhob valley ophiolite, the Khanozai and Muslim Bagh bodies contain high-Cr $(>0.6)$ chromitites while the Zhob body includes of high- $\mathrm{Al}(<0.6)$ chromitites. Chromite of high-Cr chromitite is largely uniform in composition but a few grains are partially zoned, with decrease in $\mathrm{Cr} \#$ from core to rim which reflects the melt fractionation during crystallization of chromite. In the chromite grains of high-Al chromitites, $\mathrm{Cr} \#$ and $\mathrm{FeO}_{\text {total }}$ (wt\%) decrease from rim to core which suggests that ferritchromite from high-Al chromitites had been generated by secondary processes.

The parental melt composition of high-Cr chromitites and the hydrous

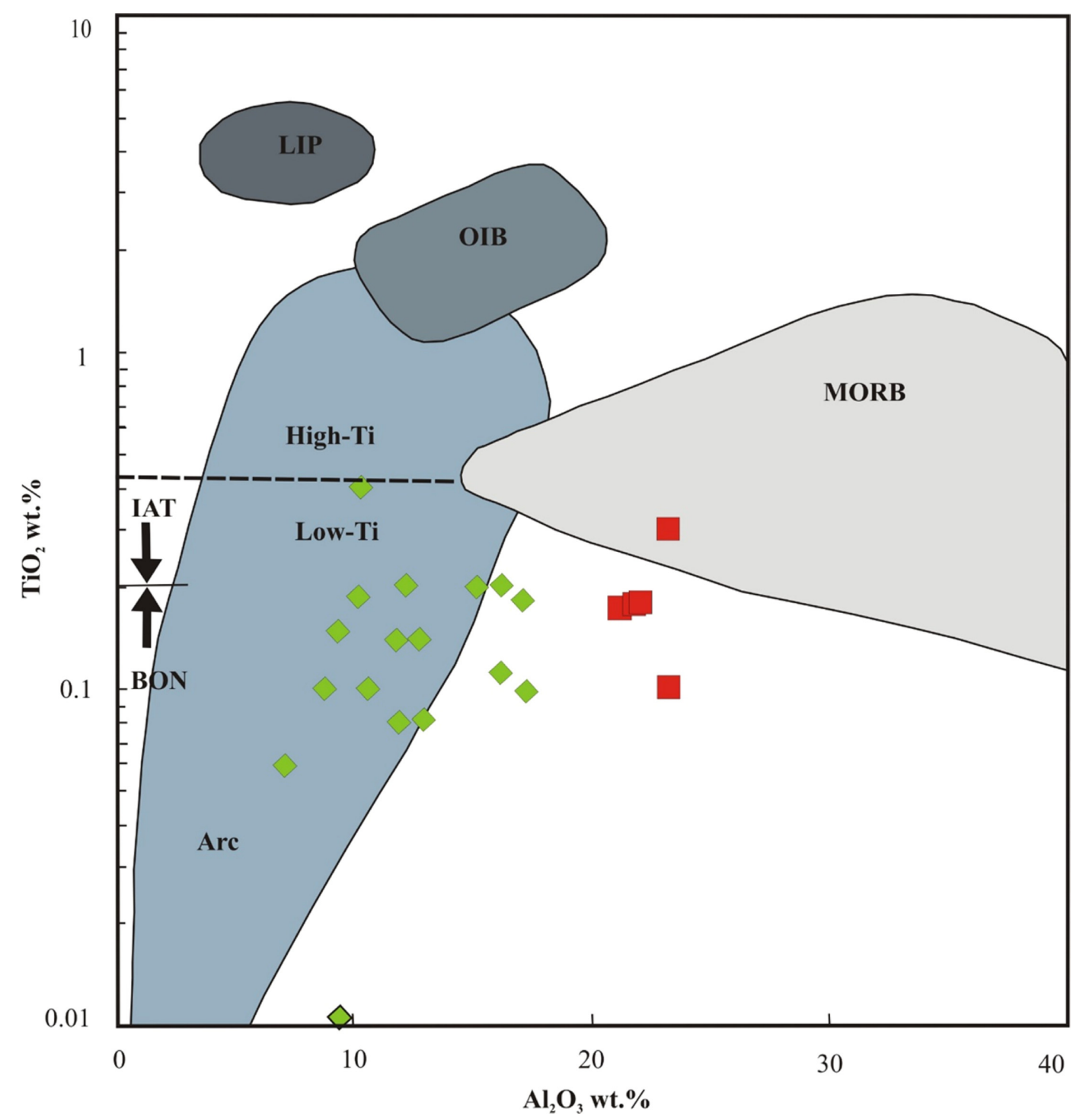

Figure 12. Tectonic discrimination using Al and Ti of chromites from chromitites of the Zhob valley ophiolite (fields after Kamenetsky et al., 2001). MORB, mid-oceanic ridge basalt; BON, boninite; LIP, large igneous province basalt; OIB, ocean island basalt; ACR, arc-related volcanic rock; IAT, island arc tholeiite. 
silicate inclusions reflect that the parental magma is boninitic, which formed by high degrees of partial melting of mantle, generating Sundersaturated and hydrous melt. In contrast, the calculated composition of parental melt of the high-Al chromitites is MORB-like melt and the presence of amphibole inclusions indicate that high-Al chromite are derived from MORB-like melt reaction with depleted mantle that produced hydrous melt.

Negative correlations of vanadium, gallium, zinc, nickel and titanium with $\mathrm{Cr} \#$ reveal the fractionation of the parental melt. The trace elements of high-Cr chromite form an identical pattern to the boninitic lava which shows that the parental melt of high-Cr is boninitic in nature. High- $\mathrm{Al}$ chromites are enriched in $\mathrm{Al}, \mathrm{Ga}, \mathrm{Ti}, \mathrm{Ni}$, and $\mathrm{Mg}$ compared to boninite and form flat patterns similar to MORB and suggest that parental melt is basaltic with MORB affinity.

The field, petrographic and geochemical data suggest that the Zhob valley chromitites are crystallized from ascending melts through a network of channels, formed by melt-rock interactions at different degrees and high-Cr chromite are crystallized out from boninite magma and may be linked to a supra-subduction zone environment, whereas high-Al chromitites are derived from MORB-like melt during reaction with depleted mantle, most probably, originated in a back arc basin tectonic environment.

\section{Acknowledgements}

This research was financially supported by the six months, HRD Foreign scholarships of the Centre of Excellence in Mineralogy, University of Baluchistan, Quetta, which was approved by the Higher Education Commission, Pakistan under its PSDP development project "Capacity Building and Strengthening of the Centre of Excellence in Mineralogy". The research was also partly supported by Higher Education Commission, Pakistan "National Research Program for Universities (NRPU) Project \# 3593" to M. Ishaq Kakar. The authors are grateful to Editor-in-Chief and reviewers for their constructive comments which improved the manuscript.

\section{References}

Ahmad, Z., 1974, Geology and Petro-chemistry of a part of the Zhob Valley Igneous Complex, Balochistan, Pakistan. Geological Survey of Pakistan Record, v. 24, pp. 1-35.

Ahmed, A.H., and Arai, S., 2003, Unexpectedly high-PGE chromitite from the deeper mantle section of the northern Oman ophiolite and its tectonic implications. Contributions to Mineralogy and Petrology, v. 143, pp. 263-278.

Ahmed, A.H., Arai, S., and Attia, A.K., 2001, Petrological characteristics of podiform chromitites and associated peridotites of the Pan African Proterozoic ophiolite complexes of Egypt. Mineralium Deposita, v. 36, p. 72-84.

Akmaz, R.M., Uysal, I., and Saka, S., 2014, Compositional variations of chromite and solid inclusions in ophiolitic chromitites from the southeastern Turkey: Implications for chromitite genesis. Ore Geology Reviews, v. 58, pp. 208-224.

Alabaster, T., Pearce, J.A., and Malpas, J., 1982, The volcanic stratigraphy and petrogenesis of the Oman ophiolite complex. Contributions to Mineralogy and Petrology, v. 81, pp. 168-183.

Arai, S., 1994, Compositional variations of olivine-chromian spinel in
Mg-rich magmas a guide to their residual spinel peridotites. Journal of Volcanology and Geothermal Research, v. 59, pp. 273-293.

Arai, S., and Abe, N., 1994, Podiform chromitite in the arc mantle: Chromitite xenoliths from the Takashima alkali basalt, southwest Japan arc. Mineralium Deposita, v. 19, pp. 434-438.

Arai, S., and Matsukage, K., 1998, Petrology of a chromitite micropod from Hess Deep, equatorial Pacific - a comparison between abyssal and Alpine type podiform chromitites. Lithos, v. 43, pp. 1-14.

Arai, S., and Yurimoto, H., 1994, Podiform chromitites of the Tari-Misaka ultramafic complex, Southwestern Japan, as mantle-melt interaction products. Economic Geology, v. 89, pp. 1279-1288.

Arai, S., Kadoshima, K., and Morishita, T., 2006, Widespread arc-related melting in the mantle section of the northern Oman ophiolite as inferred from detrital chromian spinels. Journal of Geological Society London, v. 163 , pp. 869-879.

Arai, S., Okamura, H., Kadoshima, K., Tanaka, C., Suzuki, K., and Ishimaru, S., 2011, Chemical characteristics of chromian spinel in plutonic rocks: implications for deep magma processes and discrimination of tectonic setting. Island Arc, v. 20, pp. 125-137.

Auge, T.H., 1987, Chromite deposits in the northern Oman ophiolite: Mineralogical constraints. Mineralium Deposita, v. 22, pp. 1-10.

Auzende, A.L., Guillot, G., Devouard, B., and Baronnet, A., 2006, Behavior of serpentinites in convergent context: Microstructural evidences. European Journal of Mineralogy, v. 18, pp. 21-33.

Ballhaus, C., 1998, Origin of podiform chromite deposits by magma mingling. Earth and Planetary Science Letters, v. 156, pp. 185-193.

Barnes, S.J., 2000, Chromite in komatiites, II. Modification during greenschist to mid-amphibolite facies metamorphism. Journal of Petrology, v. 41, pp. 387-409.

Barnes, S.J., and Roeder, P.L., 2001, The range of spinel compositions in terrestrial mafic and ultramafic rocks. Journal of Petrology, v. 42, pp. 2279-2302.

Bilgrami, S.A., 1963, Further data on the chemical composition of the Zhob Valley chromite. American Mineralogist, v. 48, pp. 573-587.

Bilgrami, S.A., 1964, Mineralogy and petrology of the Central part of the Hindubagh Igneous Complex, Hindu Bagh Mining District Zhob Valley, West Pakistan. Geological Survey of Pakistan Record, v. 10, pp. 28.

Bilgrami, S.A., 1969, Geology and chemical mineralogy of the Zhob Valley chromite deposits, West Pakistan. American Mineralogist, v. 54, pp. 134-148.

Bonavia, F.F., Diella, V., and Ferrario, A., 1993, Precambrian podiform chromitites from Kenticha hill, southern Ethiopia. Economic Geology, v. 88 , pp. $198-202$.

Burkhard, D.J.M., 1993, Accessory Cr-spinels: their coexistence and alteration in serpentinites. Geochimica et Cosmochimica Acta, v. 55, pp. 1297-306.

Cai, K., Sun, M., Yuan, C., Zhao, G., Xiao, W., and Long, X., 2012, Keketuohai mafic-ultramafic complex in the Chinese Altai, NW China: petrogenesis and geodynamic significance. Chemical Geology, 294 295, 26-41.

Colás, V., González-Jiménez, J.M., Griffin, W.L., Fanlo, I., Gervilla, F., O’Reilly, S.Y., Pearson, N.J., Kerestedjian, T., and Proenza, J.A., 2014, Fingerprints of metamorphism in chromite: new insights from minor and trace elements. Chemical Geology, v. 389, pp. 137-152.

Dare, S.A.S., Pearce, J.A., McDonald, I., and Styles, M.T., 2008, Tectonic discrimination of peridotites using $\mathrm{fO} 2-\mathrm{Cr} \#$ and Ga-Ti-FeIII systematics in chrome-spinel. Chemical Geology, v. 261, pp. 199-216.

Dick, H.J.B., and Bullen, T., 1984, Chromian spinel as a petrogenetic indicator in abyssal and alpine peridotite and spatially associated lavas. Contributions to Mineralogy and Petrology, v. 86, pp. 54-76.

Dönmez, C., Keskin, S., Günay, K., Çolakoğlu, A.O., Çiftçi, Y., Uysal, I., Türkel, A., and Yıldırım, N., 2014, Chromite and PGE geochemistry of the Elekdağ ophiolite (Kastamonu, northern Turkey): implications for deep magmatic processes in a supra-subduction zone setting. Ore Geology Reviews, v. 57, pp. 216-228. 
Economou-Eliopoulos, M., 2003, Apatite and Mn, Zn, Co-enriched chromite in Ni-laterites of northern Greece and their genetic significance: Journal of Geochemical Exploration, v. 80, pp. 41-54.

Gahlan, H.A., and Arai, S., 2007, Genesis of peculiarly zoned Co, Zn and Mn rich chromian spinel in serpentinite of Bou-Azzer ophiolite, AntiAtlas, Morocco. Journal Mineralogical and Petrological Sciences, v. 102, pp. 69-85.

Gansser, A., 1979, Reconnaissance visit to the Ophiolites in Balochistan. In: Farah, A., and De Jong, K.A. (Eds.), Geodynamics of Pakistan. Geological Survey of Pakistan, pp. 93-213.

Gervilla, F., Proenza, J.A., Frei, R., González-Jiménez, J.M., Garrido, C.J., Melgarejo, J.C.,Meibom, A., Díaz-martínez, R., and Lavaut, W., 2005, Distribution of platinum-group elements and Os isotopes in chromite ores from Mayarí-Baracoa Ophiolite Belt (eastern Cuba). Contributions to Mineralogy and Petrology, v. 150, pp. 589-607.

Gervilla, F., Padrón-Navarta, J.A., Kerestedjian, T., Sergeeva, I., González Jiménez, J.M., and Fanlo, I., 2012, Formation of ferrian chromite in podiform chromitites from the Golyamo Kamenyane serpentinite, Eastern Rhodopes, SE Bulgaria: a two-stage process. Contributions to Mineralogy and Petrology, v. 164, pp. 643-657.

Ghosh B., Ray J., and Morishita, T., 2014, Grain-scale plastic deformation of chromite from podiform chromitite of the Naga-Manipur ophiolite belt, India: Implication to mantle dynamics. Ore Geology Reviews, v. 56, pp. 199-208.

González-Jiménez, J.M., Kerestedjian, T., Proenza, J.A., Gervilla, F., 2009, Metamorphism on chromite ores from the Dobromirtsi Ultramafic Massif, Rhodope Mountains (SE Bulgaria). Geologica Acta, v. 7, pp. 413429.

González-Jiménez, J.M., Proenza, J.A., Gervilla, F., Melgarejo, J.C., Blanco-Moreno, J.A., Ruiz-Sanchez, R., and Griffin, W.L., 2011, High-Cr and high-Al chromitites from the Sagua de Tanamo District, MayariCristal ophiolitic massif (Eastern Cuba): constraints on their origin from mineralogy and geochemistry of chromian spinel and platinum group elements. Lithos, v. 125, pp. 101-121.

González-Jiménez, J.M., Griffin, W.L., Locmelis, M., O'Reilly, S.Y., and Pearson, N.J., 2012, LA-ICP-MS Analysis on Spinel from chromitites of different tectonic settings: Their contrasted Minor- and Trace-Elements Compositions. Abstracts, Annual V.M. Goldschmidt Conference, Montréal, Canada.

González-Jiménez, J.M., Griffin, W.L., Gervilla, F., Proenza, J.A., O'Reilly, S.Y., Pearson, and N.J., 2014b, Chromitites in ophiolites: how, where, when, why? Part I. Origin and significance of platinum-group minerals. Lithos, v. 189, pp. 127-139.

González-Jiménez, J.M., Locmelis, M., Belousova, E., Griffin, W.L., Gervilla, F., Kerestedjian, T., O'Reilly, S.Y., Pearson, N., and Sergeeva, I., 2015, Genesis and tectonic implications of podiform chromitites in the metamorphosed ultramafic massif of Dobromirtsi (Bulgaria). Gondwana Research, v. 27, pp. 555-574.

Graham, I.T., Franklin, B.J., and Marshall, B., 1996, Chemistry and mineralogy of podiform chromitite deposits, southern NSW, Australia: a guide to their origin and evolution. Mineralogy and Petrology, v. 37, pp. $129-150$.

Hajialioghli, R., Moazzen, M., Droop, G.T.R., Oberhansli, R., Bousquet, R., Jahngri, A., and Ziemann, M., 2007, Serpentine polymorphs and P$\mathrm{T}$ evolution of metaperidotites and serpentine in the Takab area, NW Iran. Mineralogical Magazine, v. 71, pp. 203-222.

Heinrich, C.A., Pettke, T., Halter, W.E., Aigner-Torres, M., Audetat, A., Gunther, D., Hattendorf, B., Bleiner, D., Guillong, M., and Horn, I., 2003, Quantitative multi-element analysis of minerals, fluid and melt inclusions by laser-ablation inductively-coupled-plasma mass-spectrometry. Geochimica et Cosmochimica Acta, v. 67, pp. 3473-3496.

Hoshino, K., and Anwar, M., 1989, Chemical composition of Chromite from the Muslim Bagh ophiolite and its implications for ore genesis: In: Okimura and Fatmi (Eds.), Tectonics and sedimentation of the Indo-Eurasian colliding plate boundary region and its influence on the mineral Developments in Pakistan. University of Hiroshima, pp. 59-68.

Hunting Survey Corporation, 1960, Reconnaissance Geology of Part of West Pakistan. A Colombo Plan cooperative project, Government of Canada, Toronto, pp. 550.

Johan, Z., and Lebel, L., 1978, Sur la genese des couches et podes de chromite dans les complexes ophiolitique. Service Géologique National BRGM, pp. 96-99.

Johan, Z., Dunlop, H., Lebel, L., Robert, J.L., and Volfinger, M., 1983, Origin of chromite deposits in ophiolitic complexes: evidence for a volatile and Na-rich reducing fluid. European Journal of Mineralogy, v. 61, pp. 105-107.

Kakar, M.I., Mahmood, K., Kerr, A.C., and Khan, M., 2013, Petrology of the Mantle Rocks from the Muslim Bagh Ophiolite, Balochistan, Pakistan, Journal of Himalayan Earth Sciences, v. 2, pp. 46.

Kakar, M.I., Kerr, A.C., Mahmood, K., Collins, A.S., Khan, M., and McDonald, I., 2014, Supra-subduction zone tectonic setting of the Muslim Bagh ophiolite, northwestern Pakistan: insights from geochemistry and petrology. Lithos, v. 202, pp. 190-206.

Kakar, M.I., Mahmood, K., Arif, M., Khan, M., Kerr, A. C., Mohibullah, M., and Kasi, A.K., 2015, Petrology and geochemistry of mafic dykes from the Muslim Bagh Ophiolite (Pakistan): implications for petrogenesis and emplacement. Turkish Journal of Earth Sciences, v. 24, pp. $165-178$.

Kamenetsky, V.S., Crawford, A.J., and Meffre, S., 2001, Factors controlling chemistry of magmatic spinel: an empirical study of associated olivine, Cr-spinel and melt inclusions from primitive rocks. Journal of Petrology, v. 42, pp. 655-671.

Kapsiotis, A., 2013, Genesis of chromitites from Korydallos, Pindos Ophiolite Complex, Greece, based on spinel chemistry and PGE-mineralogy. Journal of Geosciences, v. 58, pp. 49-69.

Kapsiotis, A., 2015, Alteration of chromitites from Voidolakkos and Xerolivado mines, Vourinos ophiolite complex, Greece: implications for deformation-induced metamorphism. Geological Journal, v. 50, pp. 739-763.

Kapsiotis, A., Ewing-Rassios, A., Uysal, I., Grieco, G., Akmaz, R.M., Saka, S., and Bussolesi, M., 2018, Compositional fingerprints of chromian spinel from the refractory chrome ores of Metalleion, Othris (Greece): implications for metallogeny and deformation of chromitites within a "hot" oceanic fault zone. Journal of Geochemical Exploration, v. 185, pp. 14-32.

Kasi, A.K., Kassi, A. M., Umar, M., Manan, R.A., and Kakar, M.I., 2012, Revised Lithostratigraphy and tectonic zones of the Pishin Belt, northwestern Pakistan. Journal of Himalayan Earth Sciences, v. 45, pp. 60.

Kelemen, P.B., 1990, Reaction between ultramafic wall rock and fractionating basaltic magma: part I, phase relations, the origin of calc-alkaline magma series, and the formation of discordant dunite. Journal of Petrology, v. 31, pp. 51-98.

Kelemen, P.B., Dick, H.J.B., and Quick, J.E., 1992, Formation of harzburgite by pervasive melt/rock interaction in the upper mantle. Nature, $\mathrm{v}$. 358, pp. 635-641.

Leblanc, M., 1995, Chromitite and ultramafic rock compositional zoning through a Paleotransform Fault, Poum, New Caledonia. Economic Geology, v. 90, pp. 2028-2039.

Leblanc, M.G., and Ceuleneer, G.S., 1992, Chromite crystallization in a multicellular magma flow: Evidence from a chromitite dike in the Oman ophiolite. Lithos, v. 2, pp. 231-257.

Leblanc, M. G., and Nicolas., 1992, Ophiolitic chromitite. International Geology Reviews, v. 34, pp. 653-686.

Lorand, J.P., and Ceuleneer, G., 1989, Silicate and base-metal sulfide inclusions in chromites from the Maqsad area (Oman ophiolite): a model for entrapment. Lithos, v. 22, pp. 173-190.

Mahmood, K., Boudier, F., Gnos, E., Monié, P., and Nicolas, A., 1995, ${ }^{40} \mathrm{Ar}{ }^{\beta 9} \mathrm{Ar}$ dating of the emplacement of the Muslim Bagh Ophiolite, Pakistan. Tectonophysics, v. 250, pp. 169-181.

Malvin, D.J., and Drake, M.J., 1987, Experimental determination of crys- 
tal/melt partitioning of $\mathrm{Ga}$ and $\mathrm{Ge}$ in the system forsterite-anorthitediopside. Geochimica et Cosmochimica Acta, v. 51, pp. 2117-2128.

Maurel, C., and Maurel, P., 1982, Etude experimentale de la distribbution de l'aluminium entre bain silicate basique et spinelle chromifere, implications petrogenetiques, Teneur en chrome des spineless. Bulletin de Mineralogie, v. 105, pp. 197-202.

Melcher, F., Grum, W., Simon, G., Thalhammer, T., and Stumpfl, E.F., 1997, Petrogenesis of the ophiolitic giant chromite deposits of Kempirsai, Kazakhstan: a study of solid and fluid inclusions in chromite. Journal of Petrology, v. 38, pp. 1419-1458.

Melcher, F., Grum, W., Thalhammer, T. V., and Thalhammer, O.A.R., 1999, The giant chromite deposits at Kempirsai, Urals: constraints from trace element (PGE, REE) and isotope data. Mineralium Deposita, v. 34, pp. 250-272.

Merlini, A., Grieco, G., and Diella, V., 2009, Ferritchromite and chromian chlorite formation in melange-hosted Kalkan chromitite (Southern Urals, Russia). American Mineralogist, v. 94, pp. 1459-1467.

Michailidis, K.M., 1990, Zoned chromites with high Mn-contents in the $\mathrm{Fe} \mathrm{Ni-Cr-laterite} \mathrm{ore} \mathrm{deposits} \mathrm{from} \mathrm{the} \mathrm{Edessa} \mathrm{area} \mathrm{in} \mathrm{northern} \mathrm{Greece.}$ Mineralium Deposita, v. 25, pp. 190-197.

Mukherjee, R., Mondal, S.K., Rosing, M.T., and Frei, R., 2010, Compositional variations in the Mesoarchean chromitites of the Nuggihalli schist belt, Western Dharwar Craton (India): potential parental melts and implications for tectonic setting. Contributions to Mineralogy and Petrology, v. 160, pp. 865-885.

Nicolas, A., 1989, Structures of ophiolites and dynamics of oceanic lithosphere. Series in Petrology and Structural Geology, Kluwer Academic Publishing, Dordrecht, v. 4, pp. 367.

Otsuki, K., Hoshino, K., Anwar, M., Mengal, J.M., Broahi, I.A., Fatmi, A.N., and Yuji, O., 1989, Breakup of Gondwanaland and emplacement of ophiolitic complexes in Muslim Bagh area of Balochistan, Pakistan. Tectonics and sedimentation of the Indo-Eurasian colliding plate boundary region and its influence on the mineral development in Pakistan, Hiroshima University, 33-57.

Pagé, P., and Barnes, S.J., 2009, Using trace elements in chromites to constrain the origin of podiform chromitites in the Thetford Mines Ophiolite, Quebec, Canada. Economic Geology, v. 104, pp. 997-1018.

Pagé, P., Barnes, S.J., Bedard, J.H., and Zientek, M.L., 2012, In situ determination of $\mathrm{Os}$, Ir, and $\mathrm{Ru}$ in chromites formed from komatiite, tholeiite and boninite magmas: implications for chromite control of Os, Ir and $\mathrm{Ru}$ during partial melting and crystal fractionation. Chemical Geology, 302-303, 3-15.

Paktunc, A.D., and Cabri, L.J., 1995, A proton- and electron-microprobe study of gallium, nickel and zinc distribution in chromian spinel. Lithos, v. 35, pp. 261-282.

Paktunc, A.D., Hulbert, L.J., Harris, D.C., 1990, Partitioning of the platinum-group and other trace elements in sulfides from the Bushveld complex and Canadian occurrences of nickel-copper sulfides. Canadian Mineralogist, v. 28, pp. 475-488.

Paraskevopoulos, G.M., and Economou, M., 1981, Zoned Mn-rich chromite from podiform type chromite ore in serpentinites of northern Greece. American Mineralogist, v. 66, pp. 1013-1019.

Paton, C., 2011, "Iolite: Freeware for the visualization and processing of mass spectrometric data". Journal of Analytical Atomic Spectrometry, v. 26, pp. 2508-2518.

Penrose (Participants), 1972, Penrose field Conference on Ophiolites, Geotime, 24-25.

Pooley, G. D., 2004, Secondary and backscattered electron imaging of weathered chromian spinel. Scanning, v. 26, pp. 240-249.

Popal, A., Kakar, M.I., Bilqees, R., Khan, M., and Ker, A.C., 2018, Geology and Petrography of Gabbroic Rocks from Khanozai Ophiolite, Northwestern Pakistan. International Research Journal of Earth Sciences, v. 7, pp. 10-22.

Proenza, J.A., Gervilla, F., Melgarejo, J.C., and Bodinier, J.L., 1999, Aland Cr-rich chromitites from the Mayarí-Baracoa Ophiolitic Belt
(Eastern Cuba): consequence of interaction between volatile-rich melts and peridotites in suprasubduction mantle. Economic Geology, v. 94, pp. 547-566.

Proenza, J. A., Ortega-Gutierrez, F., Camprubı, A., Tritlla, J., Elias-Herrera, M., and Reyes-Salas, M., 2004, Paleozoic serpentinite-enclosed chromitites from Tehuitzingo (Acatlan Complex, southern Mexico): a petrological and mineralogical study. Journal of South American Earth Sciences, v. 16, pp. 649-666.

Roberts, S.R., 1988, Ophiolitic chromitite formation: A marginal basin phenomenon. Economic Geology, v. 83, pp. 1034-1036.

Rollinson, H., 2008, The geochemistry of mantle chromitites from the northern part of the Oman ophiolite: inferred parental melt compositions. Contributions to Mineralogy and Petrology, v. 156, pp. 273-288.

Rossman, D.L., Ahmad, Z., and Abbas, S.G., 1971, Geology and Chromite deposits of the Saplai Tor Ghar and Nisai area Zhob valley complex, Hindu Bagh, Quetta Division, West Pakistan; Geological Survey of Pakistan and US Geological Survey, Interim Report, pp. 51-47.

Sawada, Y., Haq, R.M., Khan, R.S., and Aziz, A., 1992, Mesozoic igneous activity in the Muslim Bagh Area, Pakistan with special reference to the hot spot magmatism related to the breakup of Gondwanaland. Proceedings of Geoscience Colloqu., Geoscience Laboratory, Geological Survey of Pakistan, 1.

Sengor, A.M.C., 1987, Tectonics of the Tethys sides: orogenic collage development in a collisional setting. Annual Review of Earth and Planetary Sciences, v. 15, pp. 213-244.

Siddiqui, R.H., Aziz, A., Mengal, J.M., Hoshino, K., and Sawada, Y., 1996, Geology, petrochemistry and tectonic evolution of Muslim Bagh Ophiolite Complex Balochistan, Pakistan. Geoscience Laboratory, Geological Survey of Pakistan, Islamabad, v. 3, pp. 11-46.

Stowe, C.W., 1994, Compositions and tectonic settings of chromite deposits through time. Economic Geology, v. 89, pp. 528-546.

Thayer, T. P., 1964, Some critical differences between alpine-type and stratiform Peridotite gabbro complexes: International Geological Congress 21st. Copenhagen Report, Norden, v. 13, pp. 47-259.

Ulhaq, E., 2018, Petrology of Mantle Rocks from the Khanozai Ophiolites, Northern Balochistan, Pakistan. [M.Phil Thesis, University of Balochistan], 70 .

Umino, S., Yanai, S., Jaman, A.R., Nakamura, Y., and Iiyama, J.T., 1990, The transition from spreading to subduction: Evidence from the Semail Ophiolite, northern Oman mountains: Symposium "Troodos 1987", Geological Survey Department, Nicosia, Cyprus, pp. 375-384.

Uysal, I., Sadiklar, M.B., Tarkian, M., Karsli, O., and Aydin, F., 2005, Mineralogy and composition of the chromitites and their platinum-group minerals from Ortaca (Muğla-SW Turkey): evidence for ophiolitic chromitite genesis. Mineralogy and Petrology, v. 83, pp. 219-242.

Uysal, I., Tarkian, M., Sadiklar, M.B., and Sen, C., 2007a, Platinum-group elements geochemistry and mineralogy in ophiolitic chromitites from the Kop Mountains, northeastern Turkey. Canadian Mineralogist, v. 45 , pp. 355-377.

Uysal, I., Zaccarini, F., Garuti, G., Meisel, T., Tarkian, M., Bernhardt, H. J., and Sadiklar, M. B., 2007b, Ophiolitic chromitites from the Kahramanmaras area, south eastern Turkey: their platinum-group elements (PGE) geochemistry, mineralogy and Os-isotope signature. Ofioliti, v. 32, pp. 151-161.

Uysal, İ., Tarkian, M., Sadiklar, M.B., Zaccarini, F., Meisel, T., Garuti, G., and Heidrich, S., 2009, Petrology of Al- and Cr-rich ophiolitic chromitites from the Muğla, SW Turkey: implications from composition of chromite, solid inclusions of platinum-group mineral, silicate, and basemetal mineral, and Os-isotope geochemistry. Contributions to Mineralogy and Petrology, v. 158, pp. 659-674.

Uysal, I., Kapsiotis, A., Akmaz, R.M., Saka, S., and Seitz, H.M., 2018, The Guleman ophiolitic chromitites (SE Turkey) and their link to a compositionally evolving mantle source during subduction initiation. Ore Geology Reviews, v. 93, pp. 98-113.

Vrendenburg, E., 1901, A geological sketch of the Balochistan desert and 
part of eastern Persia. Memoirs of Geological Survey of India, v. 31, pp. 179-302.

Watkinson, D.H., and Mainwaring, P.R., 1980, Chromite in Ontario: geology and chromite zones, Puddy Lake-Chrome Lake area, and chromite chemistry. In: Pye, E.G. (Eds.), Geoscience Research Grant Program Summary, 1979-1980. Ontario Geological Survey, Miscellaneous Paper, v. 93, pp. 220-234.

Wilson, M., 1989, Igneous Petrogenesis. Unwin Hyman, London.

Zaccarini, F., Garuti, G., Proenza, J. A., Campos, L., Thalhammer, O.A.R, Aiglsperger, T., and Lewis, J.F., 2011, Chromite and platinum group elements mineralization in the Santa Elena Ultramafic Nappe (Costa Rica): geodynamic implications. Geologica Acta, v. 9, pp. 407-423.

Zhou, M-F., Robinson, P.T., and Bai, W.J., 1994, Formation of podiform chromitites by melt/rock interaction in the upper mantle. Mineralium Deposita, v. 29, pp. 98-101.

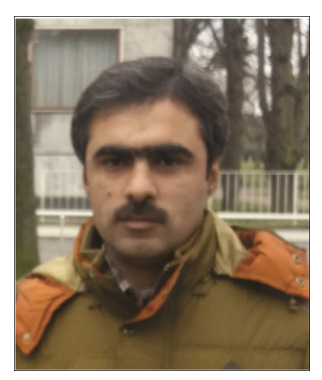

Muhammad Ayoub Khan is doing his $\mathrm{PhD}$ from Centre of Excellence in Mineralogy, University of Balochistan, Quetta, Pakistan. Currently he is serving as Assistant Professor at Centre of Excellence in Mineralogy, University of Balochistan, Quetta, Pakistan. $\mathrm{He}$ is teaching metallic minerals and doing research on economic mineral deposits associated with ophiolites.

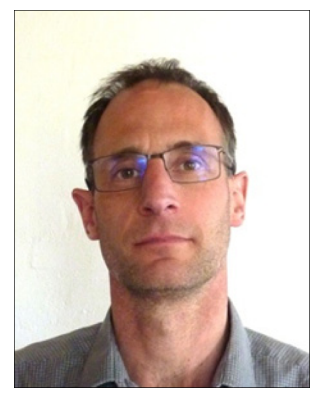

Thomas Ulrich is an Associate Professor for ore deposit research at the Department for Geoscience, Aarhus University in Denmark. He has a $\mathrm{PhD}$ from ETH Zurich in Switzerland and is conducting research in economic geology. His research involves the geochemistry of minerals and fluids in ore deposits as well as applied projects in building materials. He is a member of the Aarhus Geochemistry and Isotope Research (AGIR) group at Aarhus University where he applies in-situ trace element and isotopic analysis to unravel oreforming processes using laser ablation inductively coupled plasma mass spectrometry (LA ICP-MS).

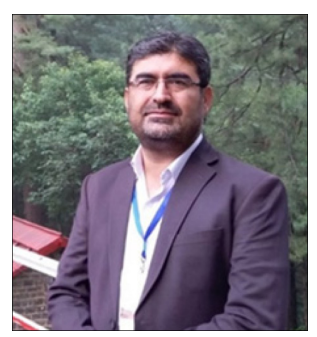

Muhammad Ishaq Kakar is an Igneous Petrologist, his research interest extends to the geology and tectonics of ophiolites and the ophiolite belts, Magmatic evolution of island arcs, Petrogenesis of hotspot related intrusive and extrusive rocks, petrology and geochemistry of mafic-ultramafic igneous rocks especially those of the ophiolite suites, the metamorphic sole rocks associated with ophiolites, and the mineralization associated with both ophiolite belts and island arcs. He is currently working as Professor and Acting Director, Centre of Excellence in Mineralogy, University of Balochistan, Quetta, Pakistan.
Zhou, M-F., Robinson, P.T., Malpas, J., and Li, Z., 1996, Podiform chromitites in the Luobusa ophiolite (southern Tibet): implications for melt-rock interaction and chromite segregation in the upper mantle. Journal of Petrology, v. 37, pp. 3-21.

Zhou, M-F., and Robinson, R.T., 1997, Origin and tectonic environment of podiform chromite deposits. Economic Geology, v. 92, pp. 259-262.

Zhou, M-F., Sun, M., Keays, R.R., and Kerrich, R.W., 1998, Controls on platinum-group elemental distributions of podiform chromitites: A case study of high-Cr and high-Al chromitites from Chinese orogenic belts: Geochimica et Cosmochimica Acta, v. 62, pp. 677-688.

Zhou, M-F, Robinson, P.T., Su B.X., Gao, J.F., Li, J.W., Yang, J.S., and Malpas, J., 2014, Compositions of chromite, associated minerals, and parental magmas of podiform chromite deposits: the role of slab contamination of asthenospheric melts in suprasubduction zone environments. Gondwana Research, v. 26, pp. 262-283.

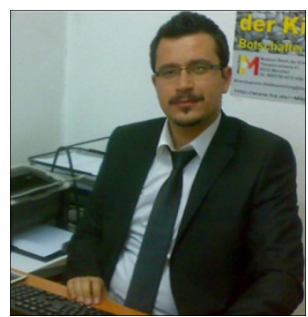

Recep Melih Akmaz is a research assistant at the University of Zonguldak Bülent Ecevit (Turkey). He completed his $\mathrm{PhD}$ degree at Karadeniz Technical University Graduate Institute of Natural and Applied Sciences (Trabzon, Turkey). His interests are mainly ophiolitic chromitites and mantle peridotites. Heresearches chromite chemistry, PGE geochemistry and mineralogy, mineral inclusions and alteration process of chromitites.

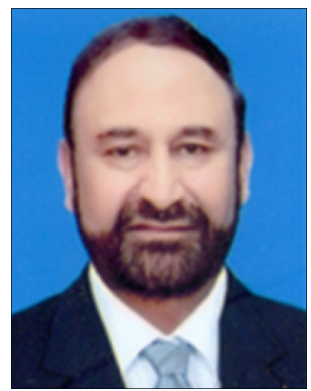

Rehanul Haq Siddiqui is affiliated as Professor in the Balochistan University of Information Technology, Engineering and Management Sciences, at Quetta, Pakistan. Before he worked as Director, in Geological Survey of Pakistan. $\mathrm{He}$ is serving as visiting Professor at Centre of Excellence in Mineralogy, University of Balochistan Quetta, Pakistan and in the China University of Geosciences at Wuhan, China.

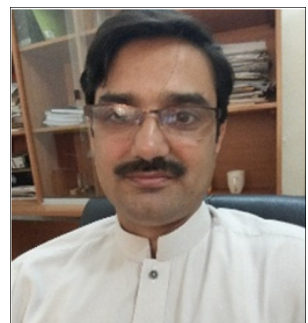

Liaqat Ali is currently working as Associate Professor at the National Centre of Excellence in Geology, University of Peshawar, Pakistan. He got his PhD from the Camborne School of Mines, University of ExeterCornwall Campus Penryn, UK. His research interest is Economic Geology, Exploration Geochemistry, GIS/RS applications to mineral deposits, Environmental Geochemistry related to mining hazards. 